\title{
ISORROPIA II: a computationally efficient thermodynamic equilibrium model for $\mathrm{K}^{+}-\mathrm{Ca}^{2+}-\mathrm{Mg}^{2+}-\mathrm{NH}_{4}^{+}-\mathrm{Na}^{+}-\mathrm{SO}_{4}^{2-}-\mathrm{NO}_{3}^{-}-\mathrm{Cl}^{-}-\mathrm{H}_{2} \mathrm{O}$ aerosols
}

\author{
C. Fountoukis ${ }^{1}$ and A. Nenes ${ }^{1,2}$ \\ ${ }^{1}$ School of Chemical and Biomolecular Engineering, Georgia Institute of Technology, 311 Ferst Drive, Atlanta, GA \\ 30332-0100, USA \\ ${ }^{2}$ School of Earth and Atmospheric Sciences, Georgia Institute of Technology, 311 Ferst Drive, Atlanta, GA 30332-0100, USA
}

Received: 24 January 2007 - Published in Atmos. Chem. Phys. Discuss.: 7 February 2007

Revised: 30 May 2007 - Accepted: 5 September 2007 - Published: 13 September 2007

\begin{abstract}
This study presents ISORROPIA II, a thermodynamic equilibrium model for the $\mathrm{K}^{+}-\mathrm{Ca}^{2+}-\mathrm{Mg}^{2+}-\mathrm{NH}_{4}^{+}-$ $\mathrm{Na}^{+}-\mathrm{SO}_{4}^{2-}-\mathrm{NO}_{3}^{-}-\mathrm{Cl}^{-}-\mathrm{H}_{2} \mathrm{O}$ aerosol system. A comprehensive evaluation of its performance is conducted against water uptake measurements for laboratory aerosol and predictions of the SCAPE2 thermodynamic module over a wide range of atmospherically relevant conditions. The two models agree well, to within $13 \%$ for aerosol water content and total PM mass, $16 \%$ for aerosol nitrate and $6 \%$ for aerosol chloride and ammonium. Largest discrepancies were found under conditions of low RH, primarily from differences in the treatment of water uptake and solid state composition. In terms of computational speed, ISORROPIA II was more than an order of magnitude faster than SCAPE2, with robust and rapid convergence under all conditions. The addition of crustal species does not slow down the thermodynamic calculations (compared to the older ISORROPIA code) because of optimizations in the activity coefficient calculation algorithm. Based on its computational rigor and performance, ISORROPIA II appears to be a highly attractive alternative for use in large scale air quality and atmospheric transport models.
\end{abstract}

\section{Introduction}

Airborne particulate matter (PM), or aerosol, play a central role in atmospheric processes. They reflect a significant amount of radiation back to space, thus enhancing the planetary albedo. Atmospheric aerosols can cause visibility impairment in highly polluted areas (Altshüller, 1984) through

Correspondence to: A. Nenes

(nenes@eas.gatech.edu) their interactions with electromagnetic radiation. By acting as cloud condensation nuclei (CCN), they affect droplet number concentration, effective radius, and reflectivity of clouds. Changes in aerosol concentrations also affect droplet size distribution, precipitation frequency and cloud lifetime. Aerosols can also be responsible for acid rain production, which can adversely affect soil and water quality, especially in environments rich in $\mathrm{SO}_{2}$ and $\mathrm{NO}_{\mathrm{x}}$. It has been established that inhaled aerosol particles are detrimental to human health, as particles can contain toxic inorganic and organic substances that are often correlated with asthma and chronic obstructive pulmonary disease (Zanobetti et al., 2000; Ramachandran and Vincent, 1999; Brauer and Brook, 1997; Schwartz, 1994). Recent studies (Kaiser, 2005) have suggested that fine particles $\left(\mathrm{PM}_{2.5}\right)$ are more effective in causing respiratory illness and premature death than larger particles due to their ability to penetrate deeper into the lung. Dockery et al. (1993), who conducted a survey on six cities over 16 years, found that people living in areas with higher aerosol concentrations had a lifespan two years less than those living in cleaner areas. The knowledge of the chemical composition and physical state of atmospheric particles may be a critical link between toxicity and particulate matter.

Atmospheric aerosols are composed of water, inorganic salts, crustal material, organics and trace metals. A large part of the particle (dry) mass is inorganic $(25-50 \%)$ with ammonium $\left(\mathrm{NH}_{4}^{+}\right)$, sodium $\left(\mathrm{Na}^{+}\right)$, sulfate $\left(\mathrm{SO}_{4}^{2-}\right)$, bisulfate $\left(\mathrm{HSO}_{4}^{-}\right)$, nitrate $\left(\mathrm{NO}_{3}^{-}\right)$and chloride $\left(\mathrm{Cl}^{-}\right)$being the most important contributors to the dry inorganic $\mathrm{PM}_{2.5}$ (Heitzenberg, 1989). Crustal species, such as $\mathrm{Ca}^{2+}, \mathrm{K}^{+}, \mathrm{Mg}^{2+}$ are a major component of dust, hence an important constituent of ambient particles. These inorganic species may be in the form of aqueous ions, or in the form of precipitated solids,

Published by Copernicus Publications on behalf of the European Geosciences Union. 
in thermodynamic equilibrium with atmospheric gases and humidity.

To compute the composition and phase state of aerosols, every atmospheric gas/aerosol model requires knowledge of the thermodynamic equilibrium state because the driving force for mass transfer of species between gas and aerosol phases is the departure from equilibrium. Performing thermodynamic equilibrium calculations for aerosol systems is a demanding computational task (e.g. Nenes et al., 1999) because it involves the optimization of a nonlinear (local) convex problem, or the solution of numerous nonlinear equations. At low relative humidity, the aqueous phase is highly concentrated (i.e. with a high ionic strength), hence strongly non-ideal and requires the use of an activity coefficient model. Therefore, efficient and accurate solution algorithms are needed.

Numerous aerosol inorganic equilibrium models have been developed, differing in the chemical species that they can treat, the solution method used and the type of input they can accept. Recent examples include AIM2 (Clegg and Pitzer, 1992; Clegg et al., 1992, 1994, 1995, 1998a, b; Wexler and Clegg, 2002), SCAPE2 (Kim et al., 1993a, b; Kim and Seinfeld, 1995; Meng et al., 1995), EQUISOLV II (Jacobson et al., 1996; Jacobson, 1999a, b), ISORROPIA (Nenes et al., 1998; Nenes et al., 1999), GFEMN (Ansari and Pandis, 1999a, b), EQSAM2 (Metzger et al., 2002a, b; Metzger et al., 2006), HETV (Makar et al., 2003), MESA (Zaveri et al., 2005a, b) and UHAERO (Amundson et al., 2006). AIM2 and GFEMN use the iterative Gibbs free energy minimization method to solve equilibrium problems for $\mathrm{NH}_{4}^{+} / \mathrm{Na}^{+} / \mathrm{NO}_{3}^{-} / \mathrm{SO}_{4}^{-2} / \mathrm{Cl}^{-}$systems. UHAERO uses the Gibbs free energy minimization method (using a primaldual method, coupled to a Newton iteration method) and offers a choice of the Pitzer, Simonson, Clegg (PSC) mole fraction-based model (Pitzer and Simonson, 1986; Clegg and Pitzer, 1992; Clegg et al., 1992) or the ExUNIQUAC model (Thomsen and Rasmussen, 1999) for the activity coefficient calculations. These models treat either the ammonium - nitrate - sulfate system or the ammonium - sodium - nitrate - chloride - sulfate system. MESA simultaneously iterates for all solid-liquid equilibria using a pseudo-transient continuation method and solves for the $\mathrm{NH}_{4}^{+} / \mathrm{Na}^{+} / \mathrm{NO}_{3}^{-} / \mathrm{SO}_{4}^{-2} / \mathrm{Cl}^{-}$system of species with the addition of calcium cations. EQUISOLV II sequentially solves for the root of each equation in the system of equilibrium reactions and then iterates over the entire domain until convergence. This method is ideal for the incorporation of new reactions and species with minimal programming effort, but optimal computational performance is obtained only on vectorized computational platforms (Zhang et al., 2000). EQSAM2 considers activity coefficients for (semi-) volatile compounds according to Metzger et al. (2002a) to solve the $\mathrm{NH}_{4}^{+} / \mathrm{Na}^{+} / \mathrm{NO}_{3}^{-} / \mathrm{SO}_{4}^{2-} / \mathrm{Cl}^{-} / \mathrm{Ca}^{2+} / \mathrm{Mg}^{2+} / \mathrm{K}^{+} / \mathrm{RCOO}^{-}$system; earlier versions of the model did not consider mineral cations and organic acids, and used parameterizated activity coefficients (Metzger et al., 1999). To express solution non-idealities, Metzger and Lelieveld (2007) suggest an approximate formulation based on osmolality and solubility measurements (instead of the activity coefficient model); this approach has been applied in the EQSAM3 model. SCAPE2 divides composition space into several subdomains based on major species that impact equilibrium partitioning and water uptake. By always attempting to solve for a liquid phase, SCAPE2 predicts the presence of water even at very low ambient relative humidities $(<10 \%)$, and for this reason often does not predict the presence of a crystalline phase (solid precipitate).

Similar to SCAPE2, ISORROPIA determines the subsystem set of equilibrium equations and solves for the equilibrium state using the chemical potential method. The code solves analytically as many equations as possible through successive substitutions; remaining equilibrium reactions are solved numerically with bisection for stability. ISORROPIA also offers the choice of using precalculated tables of binary activity coefficients and water activities of pure salt solutions, which speeds up calculations (by $\sim 30 \%$ ). Another important feature of the model is the use of mutual deliquescence of multicomponent salt particle solutions, which lowers the deliquescence point of the aerosol phase. Besides the forward problem (in which gas + aerosol concentrations of chemical species along with ambient temperature and relative humidity are used as input), ISORROPIA also offers the ability to solve for the "reverse problem", in which known quantities are the concentrations of sodium, ammonium, nitrate and sulfate in the aerosol phase together with the ambient temperature and relative humidity. The output of the reverse problem is (as in the forward problem) the concentration of species in solid, liquid and gas phase. Being computationally efficient, ISORROPIA has proved to be the model of choice for many three-dimensional air quality models (CMAQ, PMCAMx, etc.), chemical transport and general circulation models (Ansari and Pandis, 1999b; Yu et al., 2005). HETV is based on the algorithms of ISORROPIA for sulfate, nitrate and ammonium aerosol systems and is optimized for running on vectorized computational architectures.

An important limitation of the above codes (with the exception of SCAPE2, EQUISOLV II and EQSAM2,3) is lack of treatment of crustal species $(\mathrm{Ca}, \mathrm{K}, \mathrm{Mg})$. It has been shown that the consideration of crustal material in predicting the partitioning of nitrate and ammonium, especially in areas where dust comprises a significant portion of total PM, is of great importance and can considerably improve model predictions (Ansari and Pandis, 1999; Moya et al., 2002). An attempt to treat crustal species as "equivalent sodium" was met with modest success (Moya et al., 2001a), provided that $\mathrm{Ca}$ was a relatively small fraction of aerosol dry mass. Moya et al. (2002) showed that including crustal species is important in determining the aerosol size distribution, while San Martini et al. (2005) showed that treating crustal species as "equivalent" sodium may affect the predicted response of 
inorganic PM to changes in precursor concentrations. Often, crustal species are linked with inorganic anions; organic acids can be associated as well, especially with potassium in biomass burning aerosol (Trebs et al., 2005; Metzger et al., 2006). It has been shown that including both crustal species and organic acids can be important under some conditions (Metzger et al., 2006). The concentration of organic acids and their impact on global aerosol thermodynamics however still remains highly uncertain.

In the current study, we present a new model, "ISORROPIA II", in which the thermodynamics of the crustal elements of calcium, potassium and magnesium have been added to the preexisting suite of components of the ISORROPIA model. The new model, combining the computational advantages of ISORROPIA with the explicit thermodynamics of crustal species, is compared against the predictions of SCAPE2, both in terms of speciation and computational requirements.

\section{Thermodynamic equilibrium calculations}

\subsection{Equilibrium constants}

In a closed (aerosol-gas phase) system composed of $i$ chemical species and $j$ reactions at constant temperature $T$, and pressure $P$, the Gibbs free energy of the system, $G$, is minimum at chemical equilibrium. This condition is equivalent to stating that the Gibbs free energy of the reactants is equal to that of the products, and can be written as (Nenes et al., 1998):

$$
\prod_{i} a_{i}^{v_{i j}}=K_{j}(T)
$$

where $a_{i}$ is the activity of species $i, v_{i j}$ is the stoichiometric coefficient of species $i$ participating in the reaction $j$, and $K_{j}$ is the equilibrium constant of the $j$-th reaction at temperature $T$,

$K_{j}(T)=\exp \left[-\frac{\sum_{i} v_{i j} \mu_{i}^{o}(T)}{R T}\right]$

where $R$ is the universal gas constant and $\mu_{i}^{o}(T)$ is the standard chemical potential of species $i$ at 1 atm pressure and temperature $T$ (in $\mathrm{K}$ ).

$K_{j}$ is a function of temperature according to the Van't Hoff equation:

$$
\frac{d \ln K(T)}{d T}=\frac{\Delta H^{o}(T)}{R T^{2}}
$$

where $\Delta H^{o}(T)$ is the enthalpy change of the reaction at temperature $T$ (Denbigh, 1981). For a small temperature range, $\Delta H^{o}(T)$ can be approximated by:

$\Delta H^{o}(T)=\Delta H^{o}\left(T_{o}\right)+\Delta c_{p}^{o}\left(T-T_{o}\right)$ where $\Delta c_{P}^{o}(T)$ is the change of molar heat capacity of products minus reactants. By substituting Eq. (4) into Eq. (3) and integrating from a reference temperature $T_{0}$ (typically at $298.15 \mathrm{~K}$ ) to $T$, we obtain:

$$
\begin{aligned}
K(T)= & K_{o} \exp \left[-\frac{\Delta H^{o}\left(T_{o}\right)}{R T_{o}}\left(\frac{T_{o}}{T}-1\right)\right. \\
& \left.-\frac{\Delta c_{p}^{o}}{R}\left(1+\ln \left(\frac{T_{o}}{T}\right)-\frac{T_{o}}{T}\right)\right]
\end{aligned}
$$

where $K_{o}$ is the equilibrium constant at $T_{o}$.

\subsection{Activity of species}

The activity of species $i, a_{i}$, if an ideal gas, is equal to its partial pressure $\left(a_{i}=p_{i}\right)$ (Seinfeld and Pandis, 1998). If $i j$ is an electrolyte species dissolved in water, $a_{i j}=m_{i}^{v_{i}} m_{j}^{v_{j}} \gamma_{i j}^{\left(v_{i}+v_{j}\right)}$, where $\gamma_{i j}$ is the mean activity coefficient of an electrolyte species $i j$ in water, $v_{i}$ and $v_{j}$ are the moles of cations and anions, respectively, released per mole of electrolyte and $m_{i}, m_{j}$ are their molalities, respectively. The activity of each solid phase species is assumed to be unity.

\subsection{Activity coefficients}

In ISORROPIA II, the multicomponent activity coefficients, $\gamma_{12}$, for each ionic pair 1-2 are computed using Bromley's formula (Bromley, 1973),

$$
\log \gamma_{12}=-A_{\gamma} \frac{z_{1} z_{2} I^{1 / 2}}{1+I^{1 / 2}}+\frac{z_{1} z_{2}}{z_{1}+z_{2}}\left[\frac{F_{1}}{z_{1}}+\frac{F_{2}}{z_{2}}\right]
$$

where $\gamma_{12}$ is the mean activity coefficient of cation 1 and anion 2 at $298.15 \mathrm{~K}, A_{\gamma}$ is the Debye-Hückel constant $\left(0.511 \mathrm{~kg}^{0.5} \mathrm{~mol}^{-0.5}\right.$ at $\left.298.15 \mathrm{~K}\right)$ and,

$$
\begin{aligned}
F_{1}= & Y_{21} \log \gamma_{12}^{o}+Y_{41} \log \gamma_{14}^{o}+Y_{61} \log \gamma_{16}^{o}+\ldots \\
& +\frac{A_{\gamma} I^{1 / 2}}{1+I^{1 / 2}}\left[z_{1} z_{2} Y_{21}+z_{1} z_{4} Y_{41}+z_{1} z_{6} Y_{61}+\ldots\right] \\
F_{2}= & X_{12} \log \gamma_{12}^{o}+X_{32} \log \gamma_{32}^{o}+X_{52} \log \gamma_{52}^{o}+\ldots \\
& +\frac{A_{\gamma} I^{1 / 2}}{1+I^{1 / 2}}\left[z_{1} z_{2} X_{12}+z_{3} z_{2} X_{32}+z_{5} z_{2} X_{52}+\ldots\right]
\end{aligned}
$$

where odd subscripts refer to cations and even subscripts refer to anions, $Y_{21}=\left(\frac{z_{1}+z_{2}}{2}\right)^{2} \frac{m_{2}}{I}, X_{12}=\left(\frac{z_{1}+z_{2}}{2}\right)^{2} \frac{m_{1}}{I}, z_{i}$ is the absolute charge of ionic species $i$, and $\gamma_{i j}^{o}$ is the mean ionic activity coefficient of the binary pair $i-j$ ("binary" activity coefficient) computed at the ionic strength of the multicomponent solution, $I=\frac{1}{2} \sum_{i} m_{i} z_{i}^{2}$.

Following the recommendations of Kim et al. (1993), binary activity coefficients, $\gamma_{12}^{o}$, are calculated using the KusikMeissner relationship (Kusik and Meissner, 1978),

$\log \gamma_{12}^{o}=z_{1} z_{2} \log \Gamma^{o}$ 
Table 1. Thermodynamic properties for all species in ISORROPIA II*.

\begin{tabular}{|c|c|c|c|}
\hline Species & $\Delta \mu_{i}^{0}(298 \mathrm{~K}), \mathrm{kJ} \mathrm{mol}^{-1}$ & $\Delta H_{f}^{o}, \mathrm{~kJ} \mathrm{~mol}^{-1}$ & $C_{p}^{o}, \mathrm{~J} \mathrm{~mol}^{-1} \mathrm{~K}^{-1}$ \\
\hline $\mathrm{Ca}\left(\mathrm{NO}_{3}\right)_{2(\mathrm{~s})}^{\mathbf{a}}$ & -1713.15 & -2132.33 & 315.65 \\
\hline $\mathrm{CaCl}_{2(\mathrm{~s})}^{\mathbf{2}}$ & -2215.6 & -2607.9 & 322.01 \\
\hline $\mathrm{CaSO}_{4(s)}$ & -1798.280 & -2022.630 & 186.020 \\
\hline $\mathbf{K H S O}_{4(s)}$ & -1031.300 & -1160.600 & 87.160 \\
\hline $\mathbf{K}_{2} \mathbf{S O}_{4(s)}$ & -1321.370 & -1437.790 & 131.460 \\
\hline $\mathrm{KNO}_{3(\mathrm{~s})}$ & -394.860 & -494.630 & 96.400 \\
\hline $\mathbf{K C l}_{(\mathrm{s})}$ & -409.140 & -434.750 & 51.300 \\
\hline $\mathrm{MgSO}_{4(\mathrm{~s})}$ & -1170.600 & -1284.900 & 96.480 \\
\hline $\mathrm{Mg}\left(\mathrm{NO}_{3}\right)_{2(\mathrm{~s})}^{\mathbf{2}}$ & -2080.3 & -2613.28 & 391.34 \\
\hline $\mathrm{MgCl}_{2(\mathrm{~s})}^{2}$ & -2114.64 & -2499.02 & 315.06 \\
\hline $\mathbf{C a}_{(\mathrm{aq})}^{2+}$ & $-\mathbf{5 5 3 . 5 8 0}$ & -542.830 & - \\
\hline $\mathbf{K}_{(\mathrm{aq})}^{+}$ & -283.270 & -252.380 & 21.800 \\
\hline $\mathbf{M g}_{(\mathrm{aq})}^{2+}$ & -454.800 & -466.850 & - \\
\hline $\mathrm{NaCl}_{(\mathrm{s})}$ & -384.138 & -411.153 & 50.500 \\
\hline $\mathrm{NaNO}_{3(\mathrm{~s})}$ & -367.000 & -467.850 & 92.880 \\
\hline $\mathrm{Na}_{2} \mathrm{SO}_{4(\mathrm{~s})}$ & -1270.160 & -1387.080 & 128.200 \\
\hline $\mathrm{NaHSO}_{4(\mathrm{~s})}$ & -992.800 & -1125.500 & 85.000 \\
\hline $\mathrm{NH}_{4} \mathrm{Cl}_{(\mathrm{s})}$ & -202.870 & -314.430 & 84.100 \\
\hline $\mathrm{NH}_{4} \mathrm{NO}_{3}(\mathrm{~s})$ & -183.870 & -365.560 & 139.300 \\
\hline$\left(\mathrm{NH}_{4}\right)_{2} \mathrm{SO}_{4(\mathrm{~s})}$ & -901.670 & -1180.850 & 187.490 \\
\hline $\mathrm{NH}_{4} \mathrm{HSO}_{4}(\mathrm{~s})$ & -823.000 & -1026.960 & 127.500 \\
\hline$\left(\mathrm{NH}_{4}\right)_{3} \mathrm{H}\left(\mathrm{SO}_{4}\right)_{2(\mathrm{~s})}$ & -1730.000 & -2207.000 & 315.000 \\
\hline $\mathrm{HNO}_{3}(\mathrm{~g})$ & -74.720 & -135.060 & 53.350 \\
\hline $\mathrm{HCl}_{(\mathrm{g})}$ & -95.299 & -92.307 & 29.126 \\
\hline $\mathrm{NH}_{3(\mathrm{~g})}$ & -16.450 & -46.110 & 35.060 \\
\hline $\mathrm{NH}_{3(\mathrm{aq})}$ & -26.500 & -80.290 & 79.900 \\
\hline $\mathrm{H}_{(\mathrm{aq})}^{+}$ & 0.000 & 0.000 & 0.000 \\
\hline $\mathrm{Na}_{(\mathrm{aq})}^{+}$ & -261.905 & -240.120 & 46.400 \\
\hline $\mathrm{NH}_{4(\mathrm{aq})}^{+}$ & -79.310 & -132.510 & 79.900 \\
\hline $\mathrm{HSO}_{4(\mathrm{aq})}^{-}$ & -755.910 & -887.340 & -84.000 \\
\hline $\mathrm{SO}_{4(\mathrm{aq})}^{2-}$ & -744.530 & -909.270 & -293.000 \\
\hline $\mathrm{NO}_{3(\mathrm{aq})}^{-}$ & -111.250 & -207.360 & -86.600 \\
\hline $\mathrm{Cl}_{(\mathrm{aq})}^{-}$ & -131.228 & -167.159 & -136.400 \\
\hline $\mathrm{OH}_{(\mathrm{aq})}^{-}$ & -157.244 & -229.994 & -148.500 \\
\hline
\end{tabular}

* Compiled by: Kim and Seinfeld (1995) and Kim et al. (1993) unless otherwise indicated; Species in bold are new in ISORROPIA II.

* Compiled by: Kelly and Wexler (2005)

- Data not available

where

$\Gamma^{o}=\left[1+B(1+0.1 I)^{q}-B\right] \Gamma^{*}$

$B=0.75-0.065 q$

$\log \Gamma^{*}=\frac{-0.5107 I^{1 / 2}}{1+C I^{1 / 2}}$

$C=1+0.055 q \exp \left(-0.023 I^{3}\right)$

and $q$ is a parameter specific for each binary pair.
The effect of temperature on multicomponent activity coefficients is described by Meissner and Peppas (1973),

$$
\begin{aligned}
\log \gamma_{i j}(T)= & {[1.125-0.005(T-273.15)] \log \gamma_{i j}\left(T_{0}\right) } \\
& -[0.125-0.005(T-273.15)] A
\end{aligned}
$$

where $\gamma_{i j}(T)$ is the multicomponent activity coefficient of the pair of ions $i-j$ at temperature $T$, and, $A=-\frac{0.41 I^{0.5}}{1+I^{0.5}}+0.039 I^{0.92}$. 


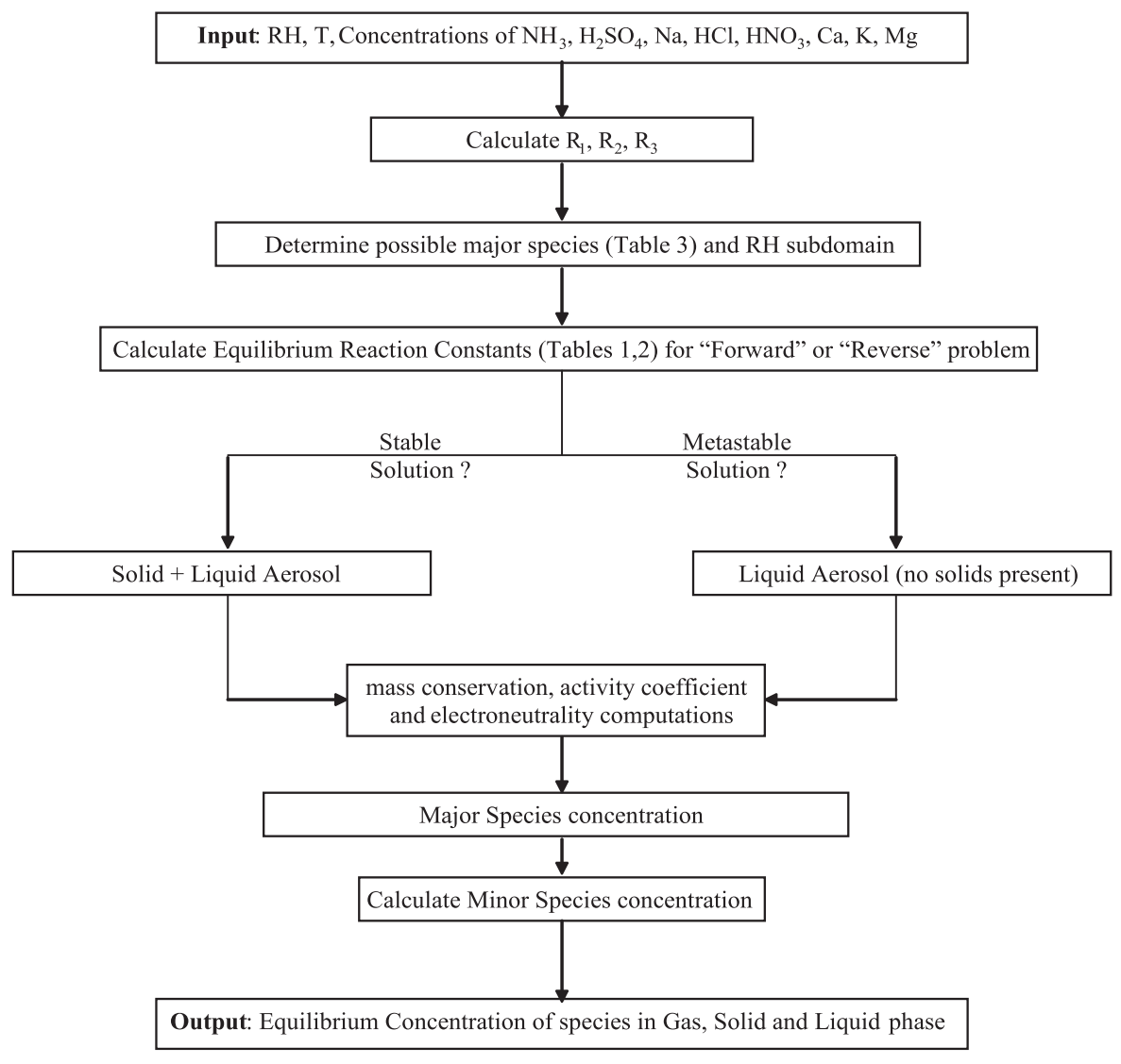

Fig. 1. Generic solution procedure of ISORROPIA II.

\subsection{Aerosol water content}

During the calculation of aerosol water content, it is assumed that aerosol curvature effects are unimportant and the ambient water vapor pressure is unaffected by the aerosol water uptake. Therefore, if ambient relative humidity is known, phase equilibrium between gas and aerosol-phase (Seinfeld and Pandis, 1998) gives that the water activity, $a_{w}$, is equal to the ambient fractional relative humidity, $\mathrm{RH}$, (i.e. expressed on a 0.0 to 1.0 scale):

$a_{w}=\mathrm{RH}$

Determining water content that satisfies the constraint of Eq. (15) from explicit calculations of water activity requires an iterative hence computationally expensive procedure (Stelson and Seinfeld, 1982). Instead, water uptake of aerosols is calculated using the ZSR correlation (Robinson and Stokes, 1965),

$W=\sum_{i} \frac{M_{i}}{m_{o i}\left(a_{w}\right)}$

where $W$ is the mass concentration of aerosol water $\left(\mathrm{kg} \mathrm{m}^{-3}\right.$ air), $M_{i}$ is the molar concentration of species $i\left(\mathrm{~mol} \mathrm{~m}^{-3}\right.$ air), and $m_{o i}\left(a_{w}\right)$ is the molality of an aqueous binary solution of the $i$-th electrolyte with the same $a_{w}$ (i.e. relative humidity) as in the multicomponent solution.

\subsection{Deliquescence relative humidity (DRH)}

For each salt, there is a characteristic relative humidity, known as the deliquescence relative humidity (DRH), above which a phase transition from solid to saturated aqueous solution occurs. The DRH varies with temperature and for small changes thereof is given by (Wexler and Seinfeld, 1991),

$\ln \frac{\operatorname{DRH}(T)}{\operatorname{DRH}\left(T_{o}\right)}=-\frac{M_{w} m_{s} L_{s}}{1000 R}\left(\frac{1}{T}-\frac{1}{T_{o}}\right)$

where $M_{w}$ is the molar mass of water and $m_{s}$ is the molality of the saturated solution at temperature $T_{o} . L_{s}$ is the latent heat of fusion for the salt from a saturated solution given by $L_{s}=\Delta H_{c r}-\Delta \mathrm{H}_{\mathrm{aq}} ; \Delta H_{c r}, \Delta \mathrm{H}_{\mathrm{aq}}$ are the molar enthalpies of formation of the crystalline phase and the species in aqueous solution, respectively. 


\subsection{Mutual deliquescence relative humidity (MDRH)}

In every multicomponent mixture there exists a characteristic relative humidity, the mutual deliquescence relative humidity, MDRH (Wexler and Seinfeld, 1991), for which all salts are simultaneously saturated with respect to all components. The MDRH is a eutectic point so it is below the DRH of all the pure solids composing the system and is the minimum $\mathrm{RH}$ for which a stable aqueous phase exists (Wexler and Seinfeld, 1991). When MDRH $<\mathrm{RH}<\mathrm{RH}_{\text {wet }}$ (where $\mathrm{RH}_{\text {wet }}$ is the DRH of the salt with the lowest DRH in the mixture under consideration) the solution is said to be in the mutual deliquescence region, MDR (Nenes et al., 1998). Computing the aerosol composition in the MDR is a computationally demanding task (e.g. Potukuchi and Wexler, 1995a, b) that we seek to avoid. Given that the MDR corresponds usually to a narrow RH range, we use the simplified approach of Nenes et al. (1998) to calculate composition in a MDR. This approach involves computing the weighted average of a "dry" and "wet" solution:

$W=(1-c) W_{\text {wet }}$

$G=c G_{\mathrm{dry}}+(1-c) G_{\mathrm{wet}}$

$S=c S_{\text {dry }}+(1-c) S_{\text {wet }}$

$D=(1-c) D_{\text {wet }}$

The weighting factor, $c$, is given by (Nenes et al., 1998):

$c=\frac{\mathrm{RH}-\mathrm{RH}_{\mathrm{wet}}}{\mathrm{MDRH}-\mathrm{RH}_{\mathrm{wet}}}$

and $G, S, D$ are the concentrations of gaseous, solid and dissolved species, respectively. The subscripts "wet" and "dry" in Eqs. (18-22) denote the two solutions which are weighted. In the above equations, we assume that gases and solids are linearly weighted according to their proximity to $\mathrm{RH}_{\text {wet }}$ and MDRH (as expressed by $c$ ), while dissolved species are scaled to the amount of water. MDRH points for the new mixtures in ISORROPIA II are shown in Table 5. Although Eqs. (18-22) are an approximation of the thermodynamic solution, they qualitatively follow the RHdependence of speciation and conserve aerosol dry mass. An assessment of this approximation done by comparing predictions of ISORROPIA II with measurements by Choi and Chan (2002) for aerosol water content of an equimolar $\mathrm{NaNO}_{3}: \mathrm{Ca}\left(\mathrm{NO}_{3}\right)_{2}$ mixture in the mutual deliquescence region. Table 6 shows the aerosol water mass fraction for $\mathrm{RH}$ ranging between 0.246 and 0.461 , which is below 0.49 , the minimum DRH of $\mathrm{Ca}\left(\mathrm{NO}_{3}\right)_{2}$ and $\mathrm{NaNO}_{3}$ (i.e. in the mutual deliquescence region). As shown in Table 6, ISORROPIA II predictions agree well with measured water mass fraction , which suggests that Eq. (18) reasonably approximates the aerosol water content in this region.

\section{ISORROPIA II: species considered and general solu- tion procedure}

The system modeled by ISORROPIA II consists of the following potential components (species in bold are new in ISORROPIA II):

Gas phase: $\mathrm{NH}_{3(\mathrm{~g})}, \mathrm{HNO}_{3(\mathrm{~g})}, \mathrm{HCl}_{(\mathrm{g})}, \mathrm{H}_{2} \mathrm{O}_{(\mathrm{g})}$

Liquid phase: $\mathrm{NH}_{4(\mathrm{aq})}+, \mathrm{Na}_{(\mathrm{aq})}^{+}, \mathrm{H}_{(\mathrm{aq})}^{+}, \mathrm{Cl}_{(\mathrm{aq})}^{-}, \mathrm{NO}_{3(\mathrm{aq})}^{-}$, $\mathrm{SO}_{4(\mathrm{aq})}^{2-}, \mathrm{HNO}_{3(\mathrm{aq})}, \mathrm{NH}_{3(\mathrm{aq})}, \mathrm{HCl}_{(\mathrm{aq})}, \mathrm{HSO}_{4(\mathrm{aq})}^{-}, \mathrm{OH}_{(\mathrm{aq})}^{-}$, $\mathrm{H}_{2} \mathrm{O}_{(\mathrm{aq})}, \mathbf{C a}_{(\mathrm{aq})}^{2+}, \mathbf{K}_{(\mathrm{aq})}^{+}, \mathbf{M g}_{(\mathrm{aq})}^{2+}$

Solid phase: $\quad\left(\mathrm{NH}_{4}\right)_{2} \mathrm{SO}_{4(\mathrm{~s})}, \quad \mathrm{NH}_{4} \mathrm{HSO}_{4(\mathrm{~s})}$, $\left(\mathrm{NH}_{4}\right)_{3} \mathrm{H}\left(\mathrm{SO}_{4}\right)_{2(\mathrm{~s})}, \quad \mathrm{NH}_{4} \mathrm{NO}_{3(\mathrm{~s})}, \quad \mathrm{NH}_{4} \mathrm{Cl}_{(\mathrm{s})}, \quad \mathrm{NaCl}_{(\mathrm{s})}$, $\mathrm{NaNO}_{3(\mathrm{~s})}, \mathrm{NaHSO}_{4(\mathrm{~s})}, \mathrm{Na}_{2} \mathrm{SO}_{4(\mathrm{~s})}, \mathrm{CaSO}_{4(\mathrm{~s})}, \mathbf{C a}\left(\mathrm{NO}_{3}\right)_{2(\mathrm{~s})}$, $\begin{array}{lllll}\mathbf{C a C l}_{2(\mathrm{~s})}, & \mathrm{K}_{2} \mathrm{SO}_{4(\mathrm{~s})}, & \mathbf{K H S O}_{4(\mathrm{~s})}, & \mathbf{K N O}_{3(s)}, & \mathbf{K C l}_{(\mathrm{s})},\end{array}$ $\mathrm{MgSO}_{4(\mathrm{~s})}, \mathrm{Mg}\left(\mathrm{NO}_{3}\right)_{2(\mathrm{~s})}, \mathrm{MgCl}_{2(\mathrm{~s})}$

where the subscripts (g), (aq), (s) denote gas, aqueous and solid, respectively. Table 1 shows thermodynamic properties for all species considered. Table 2 displays all the equilibrium reactions used in ISORROPIA II along with values for their equilibrium constants. When the concentration of crustal species $(\mathrm{Ca}, \mathrm{K}, \mathrm{Mg}$ ) is zero, routines of ISORROPIA are used, which since its original release (Nenes et al., 1998) has been substantially improved for robustness, speed and expanded to solve a wider range of problems (updates can be obtained from http://nenes.eas.gatech.edu/ISORROPIA).

\subsection{Solution procedure}

The number of species and equilibrium reactions is determined by the relative abundance of each aerosol precursor $\left(\mathrm{NH}_{3}, \mathrm{Na}, \mathrm{Ca}, \mathrm{K}, \mathrm{Mg}, \mathrm{HNO}_{3}, \mathrm{HCl}, \mathrm{H}_{2} \mathrm{SO}_{4}\right)$ and the ambient relative humidity and temperature. The major species potentially present are determined from the value of the following ratios:

$$
\begin{aligned}
& R_{1}=\frac{\left[\mathrm{NH}_{4}^{+}\right]+\left[\mathrm{Ca}^{2+}\right]+\left[\mathrm{K}^{+}\right]+\left[\mathrm{Mg}^{2+}\right]+\left[\mathrm{Na}^{+}\right]}{\left[\mathrm{SO}_{4}^{-2}\right]} \\
& R_{2}=\frac{\left[\mathrm{Ca}^{2+}\right]+\left[\mathrm{K}^{+}\right]+\left[\mathrm{Mg}^{2+}\right]+\left[\mathrm{Na}^{+}\right]}{\left[\mathrm{SO}_{4}^{-2}\right]} \\
& R_{3}=\frac{\left[\mathrm{Ca}^{2+}\right]+\left[\mathrm{K}^{+}\right]+\left[\mathrm{Mg}^{2+}\right]}{\left[\mathrm{SO}_{4}^{-2}\right]}
\end{aligned}
$$

where $[X]$ denotes the concentration of an aerosol precursor $X$ (mol m${ }^{-3}$ of air). $R_{1}, R_{2}$ and $R_{3}$ are termed "total sulfate ratio", "crustal species and sodium ratio" and "crustal species ratio" respectively; based on their values, 5 aerosol composition regimes are defined. The possible species for each regime are listed in Table 3.

The DRH at $T_{o}=298.15 \mathrm{~K}$, the thermodynamic data for the $L_{s}$ (Eq. 17) as well as the Kussik-Meissner activity coefficient parameters (Eq. 13) are shown in Table 4. Table 7 
Table 2. Equilibrium relations and temperature dependence constants used in ISORROPIA II* .

\begin{tabular}{|c|c|c|c|c|c|}
\hline Reaction & $\begin{array}{l}\text { Equilibrium } \\
\text { Constant Expression }\end{array}$ & $K^{0}(298.15 \mathrm{~K})$ & $\frac{\Delta H^{0}\left(T_{0}\right)}{R T_{0}}$ & $\frac{\Delta c_{p}^{0}}{R}$ & Units \\
\hline $\mathrm{Ca}\left(\mathrm{NO}_{3}\right)_{2(\mathrm{~s})} \leftrightarrow \mathrm{Ca}_{(\mathrm{aq})}^{2+}+2 \mathrm{NO}_{3}^{-}(\mathrm{aq})$ & {$\left[\mathrm{Ca}^{2+}\right]\left[\mathrm{NO}_{3}^{-}\right]{ }^{2} \gamma_{\mathrm{Ca}^{2+}} \gamma_{\mathrm{NO}_{3}^{-}}^{2}$} & $6.067 \times 10^{5}$ & $-11.299^{2}$ & - & $\mathrm{mol}^{3} \mathrm{~kg}^{-3}$ \\
\hline $\mathrm{CaCl}_{2(\mathrm{~s})} \leftrightarrow \mathrm{Ca}_{(\mathrm{aq})}^{2+}+2 \mathrm{Cl}_{(\mathrm{aq})}^{-}$ & {$\left[\mathrm{Ca}^{2+}\right]\left[\mathrm{Cl}^{-}\right]^{2} \gamma_{\mathrm{Ca}^{2+}} \gamma_{\mathrm{Cl}^{-}}^{2}$} & $7.974 \times 10^{11}$ & $-14.087^{\circ}$ & - & $\mathrm{mol}^{3} \mathrm{~kg}^{-3}$ \\
\hline $\mathrm{CaSO}_{4} \cdot 2 \mathrm{H}_{2} \mathrm{O}_{(\mathrm{s})} \leftrightarrow \mathrm{Ca}_{(\mathrm{aq})}^{2+}+\mathrm{SO}_{4(\mathrm{aq})}^{2-}+2 \mathrm{H}_{2} \mathrm{O}$ & {$\left[\mathrm{Ca}^{2+}\right]\left[\mathrm{SO}_{4}^{2-}\right] \gamma_{\mathrm{Ca}^{2+}} \gamma_{\mathrm{SO}_{4}^{2-}} a_{w}^{2}$} & $4.319 \times 10^{-5}$ & - & - & $\mathrm{mol}^{2} \mathrm{~kg}^{-2}$ \\
\hline $\mathrm{K}_{2} \mathrm{SO}_{4(\mathrm{~s})} \leftrightarrow 2 \mathrm{~K}_{(\mathrm{aq})}^{+}+\mathrm{SO}_{4(\mathrm{aq})}^{2-}$ & {$\left[\mathrm{K}^{+}\right]^{2}\left[\mathrm{SO}_{4}^{2-}\right] \gamma_{\mathrm{K}^{+}}^{2} \gamma_{\mathrm{SO}_{4}^{2-}}$} & $1.569 \times 10^{-2}$ & -9.589 & 45.807 & $\mathrm{~mol}^{3} \mathrm{~kg}^{-3}$ \\
\hline $\mathrm{KHSO}_{4(\mathrm{~s})} \leftrightarrow \mathrm{K}_{(\mathrm{aq})}^{+}+\mathrm{HSO}_{4(\mathrm{aq})}^{-}$ & {$\left[\mathrm{K}^{+}\right]\left[\mathrm{HSO}_{4}^{-}\right] \gamma_{\mathrm{K}^{+}} \gamma_{\mathrm{HSO}_{4}^{-}}$} & 24.016 & -8.423 & 17.964 & $\mathrm{~mol}^{2} \mathrm{~kg}^{-2}$ \\
\hline $\mathrm{KNO}_{3(\mathrm{~s})} \leftrightarrow \mathrm{K}_{(\mathrm{aq})}^{+}+\mathrm{NO}_{3(\mathrm{aq})}^{-}$ & {$\left[\mathrm{K}^{+}\right]\left[\mathrm{NO}_{3}^{-}\right] \gamma_{\mathrm{K}^{+}} \gamma_{\mathrm{NO}_{3}^{-}}$} & 0.872 & 14.075 & 19.388 & $\mathrm{~mol}^{2} \mathrm{~kg}^{-2}$ \\
\hline $\mathrm{KCl}_{(\mathrm{s})} \leftrightarrow \mathrm{K}_{(\mathrm{aq})}^{+}+\mathrm{Cl}_{(\mathrm{aq})}^{-}$ & {$\left[\mathrm{K}^{+}\right]\left[\mathrm{Cl}^{-}\right] \gamma_{\mathrm{K}^{+}} \gamma_{\mathrm{Cl}^{-}}$} & 8.680 & -6.167 & 19.953 & $\mathrm{~mol}^{2} \mathrm{~kg}^{-2}$ \\
\hline $\mathrm{MgSO}_{4(\mathrm{~s})} \leftrightarrow \mathrm{Mg}_{(\mathrm{aq})}^{2+}+\mathrm{SO}_{4(\mathrm{aq})}^{2-}$ & {$\left[\mathrm{Mg}^{2+}\right]\left[\mathrm{SO}_{4}^{2-}\right] \gamma_{\mathrm{Mg}^{2+}} \gamma_{\mathrm{SO}_{4}^{2-}}$} & $1.079 \times 10^{5}$ & 36.798 & - & $\mathrm{mol}^{2} \mathrm{~kg}^{-2}$ \\
\hline $\mathrm{Mg}\left(\mathrm{NO}_{3}\right)_{2(\mathrm{~s})} \leftrightarrow \mathrm{Mg}_{(\mathrm{aq})}^{2+}+2 \mathrm{NO}_{3}^{-}(\mathrm{aq})$ & {$\left[\mathrm{Mg}^{2+}\right]\left[\mathrm{NO}_{3}^{-}\right]^{2} \gamma_{\mathrm{Mg}^{2+}} \gamma_{\mathrm{NO}_{3}^{-}}^{2}$} & $2.507 \times 10^{15}$ & $-8.754^{\circ}$ & - & $\mathrm{mol}^{3} \mathrm{~kg}^{-3}$ \\
\hline $\mathrm{MgCl}_{2(\mathrm{~s})} \leftrightarrow \mathrm{Mg}_{(\mathrm{aq})}^{2+}+2 \mathrm{Cl}_{(\mathrm{aq})}^{-}$ & {$\left[\mathrm{Mg}^{2+}\right]\left[\mathrm{Cl}^{-}\right]^{2} \gamma_{\mathrm{Mg}^{2+}} \gamma_{\mathrm{Cl}^{-}}^{2}$} & $9.557 \times 10^{21}$ & $-1.347^{*}$ & - & $\mathrm{mol}^{3} \mathrm{~kg}^{-3}$ \\
\hline $\mathrm{HSO}_{4(\mathrm{aq})}^{-} \leftrightarrow \mathrm{H}_{(\mathrm{aq})}^{+}+\mathrm{SO}_{4(\mathrm{aq})}^{2-}$ & $\frac{\left[\mathrm{H}^{+}\right]\left[\mathrm{SO}_{4}^{2-}\right]}{\left[\mathrm{HSO}_{4}^{-}\right]} \frac{\gamma_{\mathrm{H}^{+}} \gamma_{\mathrm{SO}_{4}^{2-}}}{\gamma_{\mathrm{HSO}_{4}^{-}}}$ & $1.015 \times 10^{-2}$ & 8.85 & 25.14 & $\mathrm{~mol} \mathrm{~kg}^{-1}$ \\
\hline $\mathrm{NH}_{3(\mathrm{~g})} \leftrightarrow \mathrm{NH}_{3(\mathrm{aq})}$ & {$\left[\mathrm{NH}_{3(\mathrm{aq})}\right]$} & $5.764 \times 10^{1}$ & 13.79 & -5.39 & $\mathrm{~mol} \mathrm{~kg}-1 \mathrm{~atm}^{-1}$ \\
\hline $\mathrm{NH}_{3(\mathrm{aq})}+\mathrm{H}_{2} \mathrm{O}_{(\mathrm{aq})} \leftrightarrow \mathrm{NH}_{4(\mathrm{aq})}^{+}+\mathrm{OH}_{(\mathrm{aq})}^{-}$ & $\frac{\left[\mathrm{NH}_{4}^{+}\right]\left[\mathrm{OH}^{-}\right]}{\left[\mathrm{NH}_{3}(\mathrm{aq})\right] a_{w}} \frac{\gamma_{\mathrm{NH}_{4}^{+}} \gamma_{\mathrm{OH}^{-}}}{\gamma_{\mathrm{NH}_{3}(\mathrm{aq})}}$ & $1.805 \times 10^{-5}$ & -1.50 & 26.92 & $\mathrm{~mol} \mathrm{~kg}^{-1}$ \\
\hline $\mathrm{HNO}_{3(\mathrm{~g})} \leftrightarrow \mathrm{H}_{(\mathrm{aq})}^{+}+\mathrm{NO}_{3(\mathrm{aq})}^{-}$ & $\frac{\left[\mathrm{H}^{+}\right]\left[\mathrm{NO}_{3}^{-}\right]}{\mathrm{PHNO}_{3}} \gamma_{\mathrm{H}^{+}} \gamma_{\mathrm{NO}_{3}^{-}}$ & $2.511 \times 10^{6}$ & 29.17 & 16.83 & $\mathrm{~mol}^{2} \mathrm{~kg}^{-2} \mathrm{~atm}^{-1}$ \\
\hline $\mathrm{HNO}_{3(\mathrm{~g})} \leftrightarrow \mathrm{HNO}_{3(\mathrm{aq})}$ & $\frac{\left[\mathrm{HNO}_{3(\mathrm{aq})}\right]}{\left[\mathrm{P}_{\mathrm{HNO}_{3}}\right]} \gamma_{\mathrm{HNO}_{3(\mathrm{aq})}}$ & $2.1 \times 10^{5}$ & 29.17 & 16.83 & $\mathrm{~mol} \mathrm{~kg}{ }^{-1} \mathrm{~atm}^{-1}$ \\
\hline $\mathrm{HCl}_{(\mathrm{g})} \leftrightarrow \mathrm{H}_{(\mathrm{aq})}^{+}+\mathrm{Cl}_{(\mathrm{aq})}^{-}$ & $\frac{\left[\mathrm{H}^{+}\right]\left[\mathrm{Cl}^{-}\right]}{\left.\mathrm{P}_{\mathrm{HCl}}\right]} \gamma_{\mathrm{H}^{+}} \gamma_{\mathrm{Cl}^{-}}$ & $1.971 \times 10^{6}$ & 30.20 & 19.91 & $\mathrm{~mol}^{2} \mathrm{~kg}^{-2} \mathrm{~atm}^{-1}$ \\
\hline $\mathrm{HCl}_{(\mathrm{g})} \leftrightarrow \mathrm{HCl}_{(\mathrm{aq})} \diamond$ & $\frac{\left[\mathrm{HCl}_{(\mathrm{aq})}\right]}{\left[\mathrm{P}_{\mathrm{HCl}}\right]} \gamma_{\mathrm{HCl}(\mathrm{aq})}$ & $2.5 \times 10^{3}$ & 30.20 & 19.91 & $\mathrm{~mol} \mathrm{~kg}^{-1} \mathrm{~atm}^{-1}$ \\
\hline $\mathrm{H}_{2} \mathrm{O}_{(\mathrm{aq})} \leftrightarrow \mathrm{H}_{(\mathrm{aq})}^{+}+\mathrm{OH}_{(\mathrm{aq})}^{-}$ & $\frac{\left[\mathrm{H}^{+}\right]\left[\mathrm{OH}^{-}\right]}{a_{w}} \gamma_{\mathrm{H}^{+}} \gamma_{\mathrm{OH}^{-}}$ & $1.010 \times 10^{-14}$ & -22.52 & 26.92 & $\mathrm{~mol}^{2} \mathrm{~kg}^{-2}$ \\
\hline $\mathrm{Na}_{2} \mathrm{SO}_{4(\mathrm{~s})} \leftrightarrow 2 \mathrm{Na}_{(\mathrm{aq})}^{+}+\mathrm{SO}_{4(\mathrm{aq})}^{2-}$ & {$\left[\mathrm{Na}^{+}\right]^{2}\left[\mathrm{SO}_{4}^{2-}\right] \gamma_{\mathrm{Na}^{+}}^{2} \gamma_{\mathrm{SO}_{4}^{2-}}$} & $4.799 \times 10^{-1}$ & 0.98 & 39.75 & $\mathrm{~mol}^{3} \mathrm{~kg}^{-3}$ \\
\hline$\left(\mathrm{NH}_{4}\right)_{2} \mathrm{SO}_{4(\mathrm{~s})} \leftrightarrow 2 \mathrm{NH}_{4(\mathrm{aq})}^{+}+\mathrm{SO}_{4(\mathrm{aq})}^{2-}$ & {$\left[\mathrm{NH}_{4}^{+}\right]^{2}\left[\mathrm{SO}_{4}^{2-}\right] \gamma_{\mathrm{NH}_{4}^{+}}^{2} \gamma_{\mathrm{SO}_{4}^{2-}}$} & $1.817 \times 10^{0}$ & -2.65 & 38.57 & $\mathrm{~mol}^{3} \mathrm{~kg}^{-3}$ \\
\hline $\mathrm{NH}_{4(\mathrm{~s})} \mathrm{Cl} \leftrightarrow \mathrm{NH}_{3(\mathrm{~g})}+\mathrm{HCl}_{(\mathrm{g})}$ & $\mathrm{P}_{\mathrm{NH}_{3}} \mathrm{P}_{\mathrm{HCl}}$ & $1.086 \times 10^{-16}$ & -71.00 & 2.40 & $\mathrm{~atm}^{2}$ \\
\hline $\mathrm{NaNO}_{3(\mathrm{~s})} \leftrightarrow \mathrm{Na}_{(\mathrm{aq})}^{+}+\mathrm{NO}_{3(\mathrm{aq})}^{-}$ & {$\left[\mathrm{Na}^{+}\right]\left[\mathrm{NO}_{3}^{-}\right] \gamma_{\mathrm{Na}} \gamma_{\mathrm{NO}_{3}^{-}}$} & $1.197 \times 10^{1}$ & -8.22 & 16.01 & $\mathrm{~mol}^{2} \mathrm{~kg}^{-2}$ \\
\hline $\mathrm{NaCl}_{(\mathrm{s})} \leftrightarrow \mathrm{Na}_{(\mathrm{aq})}^{+}+\mathrm{Cl}_{(\mathrm{aq})}^{-}$ & {$\left[\mathrm{Na}^{+}\right]\left[\mathrm{Cl}^{-}\right] \gamma_{\mathrm{Na}^{+}} \gamma_{\mathrm{Cl}^{-}}$} & $3.766 \times 10^{1}$ & -1.56 & 16.90 & $\mathrm{~mol}^{2} \mathrm{~kg}^{-2}$ \\
\hline $\mathrm{NaHSO}_{4(\mathrm{~s})} \leftrightarrow \mathrm{Na}_{(\mathrm{aq})}^{+}+\mathrm{HSO}_{4(\mathrm{aq})}^{-}$ & {$\left[\mathrm{Na}^{+}\right]\left[\mathrm{HSO}_{4}^{-}\right] \gamma_{\mathrm{Na}}+\gamma_{\mathrm{HSO}_{4}^{-}}$} & $2.413 \times 10^{4}$ & 0.79 & 14.75 & $\mathrm{~mol}^{2} \mathrm{~kg}^{-2}$ \\
\hline $\mathrm{NH}_{4} \mathrm{NO}_{3(\mathrm{~s})} \leftrightarrow \mathrm{NH}_{3(\mathrm{~g})}+\mathrm{HNO}_{3(\mathrm{~g})}$ & $\mathrm{P}_{\mathrm{NH}_{3}} \mathrm{P}_{\mathrm{HNO}_{3}}$ & $4.199 \times 10^{-17}$ & -74.735 & 6.025 & $\mathrm{~atm}^{2}$ \\
\hline $\mathrm{NH}_{4} \mathrm{HSO}_{4(\mathrm{~s})} \leftrightarrow \mathrm{NH}_{4(\mathrm{aq})}^{+}+\mathrm{HSO}_{4(\mathrm{aq})}^{-}$ & {$\left[\mathrm{NH}_{4}^{+}\right]\left[\mathrm{HSO}_{4}^{-}\right] \gamma_{\mathrm{NH}_{4}^{+}} \gamma_{\mathrm{HSO}_{4}^{-}}$} & $1.383 \times 10^{0}$ & -2.87 & 15.83 & $\mathrm{~mol}^{2} \mathrm{~kg}^{-2}$ \\
\hline $\begin{array}{l}\left(\mathrm{NH}_{4}\right)_{3} \mathrm{H}\left(\mathrm{SO}_{4}\right)_{2(\mathrm{~s})} \\
\quad 3 \mathrm{NH}_{4(\mathrm{aq})}^{+}+\mathrm{HSO}_{4(\mathrm{aq})}^{-}+\mathrm{SO}_{4(\mathrm{aq})}^{2-}\end{array}$ & $\begin{array}{l}{\left[\mathrm{NH}_{4}^{+}\right]^{3}\left[\mathrm{SO}_{4}^{2-}\right]\left[\mathrm{HSO}_{4}^{-}\right] \times} \\
\gamma_{\mathrm{NH}_{4}^{+}}^{3} \gamma_{\mathrm{SO}_{4}^{2-}} \gamma_{\mathrm{HSO}_{4}^{-}}\end{array}$ & $2.972 \times 10^{1}$ & -5.19 & 54.40 & $\mathrm{~mol}^{5} \mathrm{~kg}^{-5}$ \\
\hline
\end{tabular}

* Compiled by: Kim and Seinfeld (1995) and Kim et al. (1993) unless otherwise indicated; Reactions with constants in bold are new in ISORROPIA II.

* Compiled by: Kelly and Wexler (2005)

- The equilibrium constant $K_{1 b}$ of the reaction $\mathrm{HNO}_{3(\mathrm{aq})} \stackrel{\mathrm{K}_{1 \mathrm{~b}}}{\longleftrightarrow} \mathrm{H}_{(\mathrm{aq})}^{+}+\mathrm{NO}_{3(\mathrm{aq})}^{-}$is calculated from $K_{1}$ and $K_{1 a}$ of the reactions $\mathrm{HNO}_{3(\mathrm{~g})} \stackrel{\mathrm{K}_{1}}{\longleftrightarrow} \mathrm{H}_{(\mathrm{aq})}^{+}+\mathrm{NO}_{3(\mathrm{aq})}^{-}$and $\mathrm{HNO}_{3(\mathrm{~g})} \stackrel{\mathrm{K}_{1 \mathrm{a}}}{\longleftrightarrow} \mathrm{HNO}_{3(\mathrm{aq})}$, respectively: $K_{1 b}=K_{1} / K_{1 a}$

$\diamond$ The equilibrium constant $K_{2 b}$ of the reaction $\mathrm{HCl}_{(\mathrm{aq})} \stackrel{\mathrm{K}_{2 \mathrm{~b}}}{\longleftrightarrow} \mathrm{H}_{(\mathrm{aq})}^{+}+\mathrm{Cl}_{(\mathrm{aq})}^{-}$is calculated from $K_{2}$ and $K_{2 a}$ of the reactions $\mathrm{HCl}_{(\mathrm{g})} \stackrel{\mathrm{K}_{2}}{\longleftrightarrow} \mathrm{H}_{(\mathrm{aq})}^{+}+\mathrm{Cl}_{(\mathrm{aq})}^{-}$and $\mathrm{HCl}_{(\mathrm{g})} \stackrel{\mathrm{K}_{2 \mathrm{a}}}{\longleftrightarrow} \mathrm{HCl}_{(\mathrm{aq})}$, respectively: $K_{2 b}=K_{2} / K_{2 a}$

- Data not available 
Table 3. Potential species for the five aerosol types ${ }^{\mathrm{a}}$.

\begin{tabular}{|c|c|c|c|c|c|c|c|}
\hline$R_{1}$ & $R_{2}$ & $R_{3}$ & Aerosol type & Solid phase & $\begin{array}{l}\text { Major species } \\
\text { Aqueous phase }\end{array}$ & Gas phase & Minor species \\
\hline$R_{1}<1$ & any value & any value & $\begin{array}{l}\text { Sulfate Rich } \\
\text { (free acid) }\end{array}$ & $\begin{array}{l}\mathrm{NaHSO}_{4}, \quad \mathrm{NH}_{4} \mathrm{HSO}_{4}, \\
\text { KHSO }_{4}, \mathbf{C a S O}_{4}\end{array}$ & $\begin{array}{l}\mathrm{Na}^{+}, \mathrm{NH}_{4}^{+}, \mathrm{H}^{+}, \mathrm{HSO}_{4}^{-}, \\
\mathrm{SO}_{4}^{2-}, \mathrm{NO}_{3}^{-}, \mathrm{Cl}^{-}, \mathbf{C a}^{2+}, \\
\mathbf{K}^{+}, \mathrm{H}_{2} \mathrm{O}\end{array}$ & $\mathrm{H}_{2} \mathrm{O}$ & $\begin{array}{l}\mathrm{NH}_{3(\mathrm{~g})}, \\
\mathrm{NO}_{3(\mathrm{aq})}^{-}, \\
\mathrm{Cl}_{(\mathrm{aq}}^{-}, \\
\mathrm{NH}_{3(\mathrm{aq})}, \\
\mathrm{HNO}_{3(\mathrm{aq})},\end{array}$ \\
\hline $1 \leq R_{1}<2$ & any value & any value & Sulfate Rich & $\begin{array}{l}\mathrm{NaHSO}_{4}, \quad \mathrm{NH}_{4} \mathrm{HSO}_{4}, \\
\mathrm{Na}_{2} \mathrm{SO}_{4}, \quad\left(\mathrm{NH}_{4}\right)_{2} \mathrm{SO}_{4}, \\
\left(\mathrm{NH}_{4}\right)_{3} \mathrm{H}\left(\mathrm{SO}_{4}\right)_{2}, \\
\mathbf{C a S O}_{4}, \quad \mathbf{K H S O}_{4}, \\
\mathbf{K}_{2} \mathbf{S O}_{4}, \mathbf{M g S O}_{4}\end{array}$ & $\begin{array}{l}\mathrm{Na}^{+}, \mathrm{NH}_{4}^{+}, \mathrm{H}^{+}, \mathrm{HSO}_{4}^{-}, \\
\mathrm{SO}_{4}^{2-}, \mathrm{NO}_{3}^{-}, \mathrm{Cl}^{-}, \mathrm{Ca}^{2+}, \\
\mathbf{K}^{+}, \mathbf{M g}^{2+}, \mathrm{H}_{2} \mathrm{O}\end{array}$ & $\mathrm{H}_{2} \mathrm{O}$ & $\begin{array}{l}\mathrm{NH}_{3(\mathrm{~g})}, \\
\mathrm{NO}_{3(\mathrm{aq})}^{-}, \\
\mathrm{Cl}_{(\mathrm{aq})}^{-}, \\
\mathrm{NH}_{3(\mathrm{aq})}, \\
\mathrm{HNO}_{3(\mathrm{aq})}, \\
\mathrm{HCl}_{(\mathrm{aq})}\end{array}$ \\
\hline$R_{1} \geq 2$ & $R_{2}<2$ & any value & $\begin{array}{l}\text { Sulfate Poor, } \\
\text { Crustal \& Sodium } \\
\text { Poor }\end{array}$ & $\begin{array}{lr}\mathrm{Na}_{2} \mathrm{SO}_{4}, & \left(\mathrm{NH}_{4}\right)_{2} \mathrm{SO}_{4}, \\
\mathrm{NH}_{4} \mathrm{NO}_{3}, & \mathrm{NH}_{4} \mathrm{Cl}, \\
\mathbf{C a S O}_{4}, \mathbf{K}_{2} \mathbf{S O}_{4}, \mathbf{M g S O}_{4}\end{array}$ & $\begin{array}{l}\mathrm{Na}^{+}, \mathrm{NH}_{4}^{+}, \mathrm{H}^{+}, \mathrm{SO}_{4}^{2-}, \\
\mathrm{NO}_{3}^{-}, \mathrm{Cl}^{-}, \mathbf{C a}^{2+}, \mathbf{K}^{+}, \\
\mathbf{M g}^{2+}, \mathrm{H}_{2} \mathrm{O}, \mathrm{NH}_{3(\mathrm{aq})}, \\
\mathrm{HNO}_{3(\mathrm{aq})}, \mathrm{HCl}_{(\mathrm{aq})}\end{array}$ & $\begin{array}{l}\mathrm{HNO}_{3}, \quad \mathrm{HCl}, \\
\mathrm{NH}_{3}, \mathrm{H}_{2} \mathrm{O}\end{array}$ & $\mathrm{HSO}_{4(\mathrm{aq})}^{-}$ \\
\hline$R_{1} \geq 2$ & $R_{2} \geq 2$ & $R_{3}<2$ & $\begin{array}{l}\text { Sulfater Poor, } \\
\text { Crustal \& Sodium } \\
\text { Rich, Crustal Poor }\end{array}$ & $\begin{array}{l}\mathrm{Na}_{2} \mathrm{SO}_{4}, \mathrm{NaNO}_{3}, \mathrm{NaCl}, \\
\mathrm{NH}_{4} \mathrm{NO}_{3}, \quad \mathrm{NH}_{4} \mathrm{Cl}, \\
\mathbf{C a S O}_{4}, \mathbf{K}_{2} \mathbf{S O}_{4}, \mathbf{M g S O}_{4}\end{array}$ & $\begin{array}{l}\mathrm{Na}^{+}, \mathrm{NH}_{4}^{+}, \mathrm{H}^{+}, \mathrm{SO}_{4}^{2-}, \\
\mathrm{NO}_{3}^{-}, \mathrm{Cl}^{-}, \mathbf{C a}^{2+}, \mathbf{K}^{+}, \\
\mathbf{M g}^{2+}, \mathrm{H}_{2} \mathrm{O}, \mathrm{NH}_{3(\mathrm{aq})}, \\
\mathrm{HNO}_{3(\mathrm{aq})}, \mathrm{HCl}_{(\mathrm{aq})}\end{array}$ & $\begin{array}{l}\mathrm{HNO}_{3}, \quad \mathrm{HCl}, \\
\mathrm{NH}_{3}, \mathrm{H}_{2} \mathrm{O}\end{array}$ & $\mathrm{HSO}_{4(\mathrm{aq})}^{-}$ \\
\hline$R_{1} \geq 2$ & $R_{2} \geq 2$ & $R_{3}>2$ & $\begin{array}{l}\text { Sulfate Poor, } \\
\text { Crustal \& Sodium } \\
\text { Rich, Crustal Rich }\end{array}$ & $\begin{array}{l}\mathrm{NaNO}_{3}, \mathrm{NaCl}, \mathrm{NH}_{4} \mathrm{NO}_{3}, \\
\mathrm{NH}_{4} \mathrm{Cl}, \mathbf{C a S O} \mathbf{C}_{4}, \mathbf{K}_{2} \mathbf{S O}_{4}, \\
\mathbf{M g S O}_{4}, \quad \mathbf{C a}\left(\mathbf{N O}_{3}\right)_{2}, \\
\mathbf{C a C l}_{2}, \quad \mathbf{M g}\left(\mathbf{N O}_{3}\right)_{2}, \\
\mathbf{M g C l}_{2}, \mathbf{K N O} \mathbf{K}_{3}, \mathbf{K C l}\end{array}$ & $\begin{array}{l}\mathrm{Na}^{+}, \mathrm{NH}_{4}^{+}, \mathrm{H}^{+}, \mathrm{SO}_{4}^{2-}, \\
\mathrm{NO}_{3}^{-}, \mathrm{Cl}^{-}, \mathbf{C a}^{2+}, \quad \mathbf{K}^{+}, \\
\mathbf{M g}^{2+}, \quad \mathrm{H}_{2} \mathrm{O}, \quad \mathrm{NH}_{3(\mathrm{aq})}, \\
\mathrm{HNO}_{3(\mathrm{aq})}, \mathrm{HCl}_{(\mathrm{aq})}\end{array}$ & $\begin{array}{l}\mathrm{HNO}_{3}, \quad \mathrm{HCl}, \\
\mathrm{NH}_{3}, \mathrm{H}_{2} \mathrm{O}\end{array}$ & $\mathrm{HSO}_{4(\mathrm{aq})}^{-}$ \\
\hline
\end{tabular}

a Species in bold are new in ISORROPIA II

displays the polynomial fit parameters for computing the molalities of binary solutions as a function of water activity (obtained from Kim and Seinfeld, 1995; Ha and Chan, 1999; Kelly and Wexler, 2005, 2006) for $\mathrm{CaSO}_{4}, \mathrm{Ca}\left(\mathrm{NO}_{3}\right)_{2}$, $\mathrm{CaCl}_{2}, \mathrm{~K}_{2} \mathrm{SO}_{4}, \mathrm{KHSO}_{4}, \mathrm{KNO}_{3}, \mathrm{KCl}, \mathrm{MgSO}_{4}, \mathrm{Mg}\left(\mathrm{NO}_{3}\right)_{2}$ and $\mathrm{MgCl}_{2}$. For $\left(\mathrm{NH}_{4}\right)_{2} \mathrm{SO}_{4}, \mathrm{NH}_{4} \mathrm{HSO}_{4},\left(\mathrm{NH}_{4}\right)_{3} \mathrm{H}\left(\mathrm{SO}_{4}\right)_{2}$, $\mathrm{NH}_{4} \mathrm{NO}_{3}, \mathrm{NH}_{4} \mathrm{Cl}, \mathrm{NaCl}, \mathrm{NaNO}_{3}, \mathrm{NaHSO}_{4}$ and $\mathrm{Na}_{2} \mathrm{SO}_{4}$, the water activity database was updated since the original release of ISORROPIA, using the output from the AIM model (http://www.hpc1.uea.ac.uk/ e770/aim.html).

As in ISORROPIA, ISORROPIA II solves two classes of problems:

1. Forward (or "closed") problems, in which known quantities are $T, \mathrm{RH}$ and the total (gas + aerosol) concentrations of $\mathrm{NH}_{3}, \mathrm{H}_{2} \mathrm{SO}_{4}, \mathrm{Na}, \mathrm{HCl}, \mathrm{HNO}_{3}, \mathrm{Ca}, \mathrm{K}$, and $\mathrm{Mg}$.

2. Reverse (or “open”) problems, in which known quantities are $T, \mathrm{RH}$ and the precursor concentrations of $\mathrm{NH}_{3}$, $\mathrm{H}_{2} \mathrm{SO}_{4}, \mathrm{Na}, \mathrm{HCl}, \mathrm{HNO}_{3}, \mathrm{Ca}, \mathrm{K}$, and $\mathrm{Mg}$ in the aerosol phase.

Below the MDRH of an aerosol mixture, the particle is a solid if the aerosol is following its deliquescence branch. However, when the RH over a wet particle is decreasing, the wet aerosol may not crystallize below the MDRH but instead remain in a metastable state, where it is composed of an aqueous supersaturated solution (e.g. Seinfeld and Pandis, 1998). ISORROPIA II can address both states (termed "stable" where salts precipitate once the aqueous phase becomes saturated with respect to them, and, "metastable", if the aerosol is composed only of an aqueous phase which can be supersaturated with respect to dissolved salts).

Depending on the three sulfate ratios, the relative humidity and the temperature, ISORROPIA II solves the appropriate set of equilibrium equations, and, together with mass conservation, electroneutrality, water activity equations and activity coefficient calculations, the final concentrations at thermodynamic equilibrium are obtained. Figure 1 illustrates a general description of the solution procedure.

\subsection{Important issues}

- When calculating species concentration, the stable state solution algorithm of ISORROPIA II starts with assuming a completely dry aerosol. As the ambient relative humidity increases (or decreases), ISORROPIA II dissolves each of the salts present (depending on their DRH) and calculates solid and ion concentrations and water uptake. The exact opposite methodology is 
adopted by SCAPE2, which initially assumes that all salts present are completely dissolved; based on the ambient relative humidity and DRH of each salt calculates solid concentration if a precipitate is assumed to form. Differences in the "solution dynamics" may lead to differences in water content and speciation, especially at low RH, and are further analyzed in Sect. 4.

- ISORROPIA II uses the principle of "compositional invariance with RH cycling" to determine the aerosol composition at low RH (i.e. when the aerosol is solid). This is done because aerosol cycles RH many times in nature throughout its lifetime and the invariant solution will better represent its composition in the atmosphere. Compositional invariance is applied when the aerosol contains volatile anions, sulfate and non-volatile univalent cations $\left(\mathrm{Na}^{+}, \mathrm{K}^{+}\right)$. In such cases, $\mathrm{Na}_{(\mathrm{aq})}$ and $\mathrm{K}_{(\mathrm{aq})}$ preferentially associate with $\mathrm{SO}_{4(\text { aq) }}$ to form $\mathrm{Na}_{2} \mathrm{SO}_{4(\mathrm{~s})}$ and $\mathrm{K}_{2} \mathrm{SO}_{4(\mathrm{~s})}$ before they are bound with $\mathrm{NO}_{3(\mathrm{aq})}$ and $\mathrm{Cl}_{(\mathrm{aq})}$ to form $\mathrm{NaNO}_{3(\mathrm{~s})}, \mathrm{KNO}_{3(\mathrm{~s})}, \mathrm{NaCl}_{(\mathrm{s})}$, and $\mathrm{KCl}_{(\mathrm{s})}$. Other models may not adopt this approach and may lead to differences in predicted water uptake, especially at low RH. For example, in an aerosol with 2 moles of $\mathrm{K}_{(\mathrm{aq})}, 0.7$ moles of $\mathrm{SO}_{4(\mathrm{aq})}, \mathrm{NO}_{3(\mathrm{aq})}$ and $\mathrm{Cl}_{(\mathrm{aq})}$, ISORROPIA II predicts that $\mathrm{K}_{(\mathrm{aq})}$ will preferentially associate with $\mathrm{SO}_{4(\mathrm{aq})}$ to form $\mathrm{K}_{2} \mathrm{SO}_{4(\mathrm{~s})}$. The remaining $\mathrm{K}_{(\mathrm{aq})}$ will associate with $\mathrm{NO}_{3(\mathrm{aq})}$ and $\mathrm{Cl}_{(\mathrm{aq})}$ to form $\mathrm{KNO}_{3}$ and $\mathrm{KCl}$. This is because $\mathrm{SO}_{4}\left(\right.$ as $\left.\mathrm{H}_{2} \mathrm{SO}_{4}\right)$ is less volatile than $\mathrm{NO}_{3}\left(\right.$ as $\left.\mathrm{HNO}_{3}\right)$ and $\mathrm{Cl}$ (as $\left.\mathrm{HCl}\right)$ when exposed to $\mathrm{RH}$ cycling, thus $\mathrm{K}_{2} \mathrm{SO}_{4(s)}$ is more likely to form.

\subsection{Simplifications and assumptions in ISORROPIA II}

Numerous simplifying assumptions are taken to increase computational speed and numerical stability without substantially compromising rigor. These are:

- Sulfuric acid, sodium and crustal species have a very low vapor pressure and are assumed to exclusively reside in the aerosol phase.

- The first dissociation of sulfuric acid $\left(\mathrm{H}_{2} \mathrm{SO}_{4(\mathrm{aq}} \rightarrow \mathrm{H}^{+}+\mathrm{HSO}_{4}^{-}\right)$is assumed to be complete and not considered in the equilibrium calculations.

- For a wide range of ionic strengths (0-30 M), typical of ambient aerosols, the solubility product of magnesium sulfate was found to be always less than its equilibrium constant. Therefore, ISORROPIA II assumes $\mathrm{MgSO}_{4(\mathrm{~s})}$ is always deliquesced when an aqueous phase is present, avoiding any computations for precipitating $\mathrm{MgSO}_{4(\mathrm{~s})}$ out of solution.

- Calcium sulfate $\left(\mathrm{CaSO}_{4}\right)$ is assumed completely insoluble.
Table 4. Deliquescence relative humidity, temperature dependence and $q$ parameter values for all the salts modeled in ISORROPIA II ${ }^{1}$.

\begin{tabular}{|c|c|c|c|}
\hline Salt & DRH (298.15 K) & $-\frac{18}{1000 R} L_{S} m_{S}$ & $q$ \\
\hline $\mathrm{Ca}\left(\mathrm{NO}_{3}\right)_{2}$ & $0.4906^{*}$ & $509.4^{\#}$ & $0.93^{*}$ \\
\hline $\mathrm{CaCl}_{2}$ & $0.2830^{*}$ & 551.1 $^{\#}$ & $2.40^{*}$ \\
\hline $\mathrm{CaSO}_{4}$ & $0.9700^{*}$ & - &,$-^{\mathrm{a}}$ \\
\hline $\mathrm{KHSO}_{4}$ & $0.8600^{24}$ & - &,$-^{b}$ \\
\hline $\mathbf{K}_{2} \mathbf{S O}_{4}^{*}$ & 0.9751 & 35.6 & -0.25 \\
\hline $\mathrm{KNO}_{3}^{*}$ & 0.9248 & - & -2.33 \\
\hline $\mathrm{KCl}^{*^{\prime}}$ & 0.8426 & 158.9 & 0.92 \\
\hline $\mathrm{MgSO}_{4}$ & $0.8613^{\diamond}$ & $-714.5^{*}$ & $0.15^{*}$ \\
\hline $\operatorname{Mg}\left(\mathrm{NO}_{3}\right)_{2}$ & $0.5400^{\diamond}$ & $230.2^{\#}$ & $2.32^{*}$ \\
\hline $\mathbf{M g C l}_{2}$ & $0.3284^{*}$ & $\mathbf{4 2 . 2 3}^{\#}$ & $2.90^{*}$ \\
\hline $\mathrm{NaCl}^{1}$ & 0.7528 & 25.0 & 2.23 \\
\hline $\mathrm{Na}_{2} \mathrm{SO}_{4}^{\infty}$ & 0.9300 & 80.0 & -0.19 \\
\hline $\mathrm{NaNO}_{3}^{4_{4}^{4}}$ & 0.7379 & 304.0 & -0.39 \\
\hline$\left(\mathrm{NH}_{4}\right)_{2} \mathrm{SO}_{4}^{\boldsymbol{p}}$ & 0.7997 & 80.0 & -0.25 \\
\hline $\mathrm{NH}_{4} \mathrm{NO}_{3}^{\infty}$ & 0.6183 & 852.0 & -1.15 \\
\hline $\mathrm{NH}_{4} \mathrm{Cl}^{n^{3}}$ & 0.7710 & 239.0 & 0.82 \\
\hline $\mathrm{NH}_{4} \mathrm{HSO}_{4}$ & $0.4000^{\infty}$ & $384.0^{\infty}$ & $(+),{ }^{\mathrm{c}}$ \\
\hline $\mathrm{NaHSO}_{4}$ & $0.5200^{\wedge}$ & $-45.0^{\infty}$ & $(+),{ }^{\mathrm{d}}$ \\
\hline$\left(\mathrm{NH}_{4}\right)_{3} \mathrm{H}\left(\mathrm{SO}_{4}\right)_{2}$ & $0.6900^{\infty}$ & $186.0^{\infty}$ & $(+),{ }^{\mathrm{e}}$ \\
\hline $\mathrm{H}_{2} \mathrm{SO}_{4}^{*}$ & 0.000 & - & -0.1 \\
\hline $\mathrm{H}-\mathrm{HSO}_{4}^{\oplus}$ & 0.000 & - & 8.00 \\
\hline $\mathrm{HNO}_{3}^{\boldsymbol{\varphi}}$ & N/A & - & 2.60 \\
\hline $\mathrm{HCl}^{\infty}$ & N/A & - & 6.00 \\
\hline
\end{tabular}

${ }^{1}$ Species in bold are new in ISORROPIA II

* Kim and Seinfeld (1995)

\# Kelly and Wexler (2005)

* Pilinis and Seinfeld (1989)

$\diamond$ Ha and Chan (1999)

a Kim et al. (1993)

- Data not available

a $\gamma_{\mathrm{CaSO}_{4}}=0$

b $\gamma_{\mathrm{KHSO}_{4}}=\left(\frac{\gamma_{\mathrm{H}-\mathrm{HSO}_{4}} \cdot \gamma_{\mathrm{KCl}}}{\gamma_{\mathrm{HCl}}}\right)^{1 / 2}$

c $\gamma_{\mathrm{NH}_{4} \mathrm{HSO}_{4}}=\left(\frac{\gamma_{\mathrm{H}-\mathrm{HSO}_{4}} \cdot \gamma_{\mathrm{NH}_{4} \mathrm{Cl}}}{\gamma_{\mathrm{HCl}}}\right)^{1 / 2}$

d $\gamma_{\mathrm{NaHSO}_{4}}=\left(\frac{\gamma_{\mathrm{H}-\mathrm{HSO}_{4}} \cdot \gamma_{\mathrm{NaCl}}}{\gamma_{\mathrm{HCl}}}\right)^{1 / 2}$

e $\gamma_{\left(\mathrm{NH}_{4}\right)_{3} \mathrm{H}\left(\mathrm{SO}_{4}\right)_{2}}=\left(\gamma_{\left(\mathrm{NH}_{4}\right)_{2} \mathrm{SO}_{4}}^{3} \cdot \gamma_{\mathrm{NH}_{4} \mathrm{HSO}_{4}}\right)^{1 / 5}$

- For sulfate rich cases $\left(\mathrm{R}_{1}<2\right), \quad \mathrm{NH}_{3(\mathrm{~g})}, \quad \mathrm{NO}_{3(\mathrm{aq})}^{-}$ and $\mathrm{Cl}_{(\mathrm{aq})}^{-}$are assumed minor species that do not significantly perturb the equilibrium through the $\mathrm{NH}_{3(\mathrm{~g})}^{+} \mathrm{H}_{2} \mathrm{O}_{(\mathrm{aq})} \leftrightarrow \mathrm{NH}_{4(\mathrm{aq})}^{+}+\mathrm{OH}_{(\mathrm{aq})}^{-}$, $\mathrm{HNO}_{3(\mathrm{~g})} \leftrightarrow \mathrm{H}_{(\mathrm{aq})}^{+}+\mathrm{NO}_{3(\mathrm{aq})}^{-}$and $\mathrm{HCl}_{(\mathrm{g})} \leftrightarrow \mathrm{H}_{(\mathrm{aq})}^{+}+\mathrm{Cl}_{(\mathrm{aq})}^{-}$ reactions, respectively. The code solves the appropriate set of equilibrium reactions (for the major species) and then the three gases $\left(\mathrm{NH}_{3(\mathrm{~g})}, \mathrm{HNO}_{3(\mathrm{~g})}, \mathrm{HCl}_{(\mathrm{g})}\right)$ 
Table 5. Mutual deliquescence relative humidity, for the new salt mixtures modeled in ISORROPIA II ${ }^{1}$.

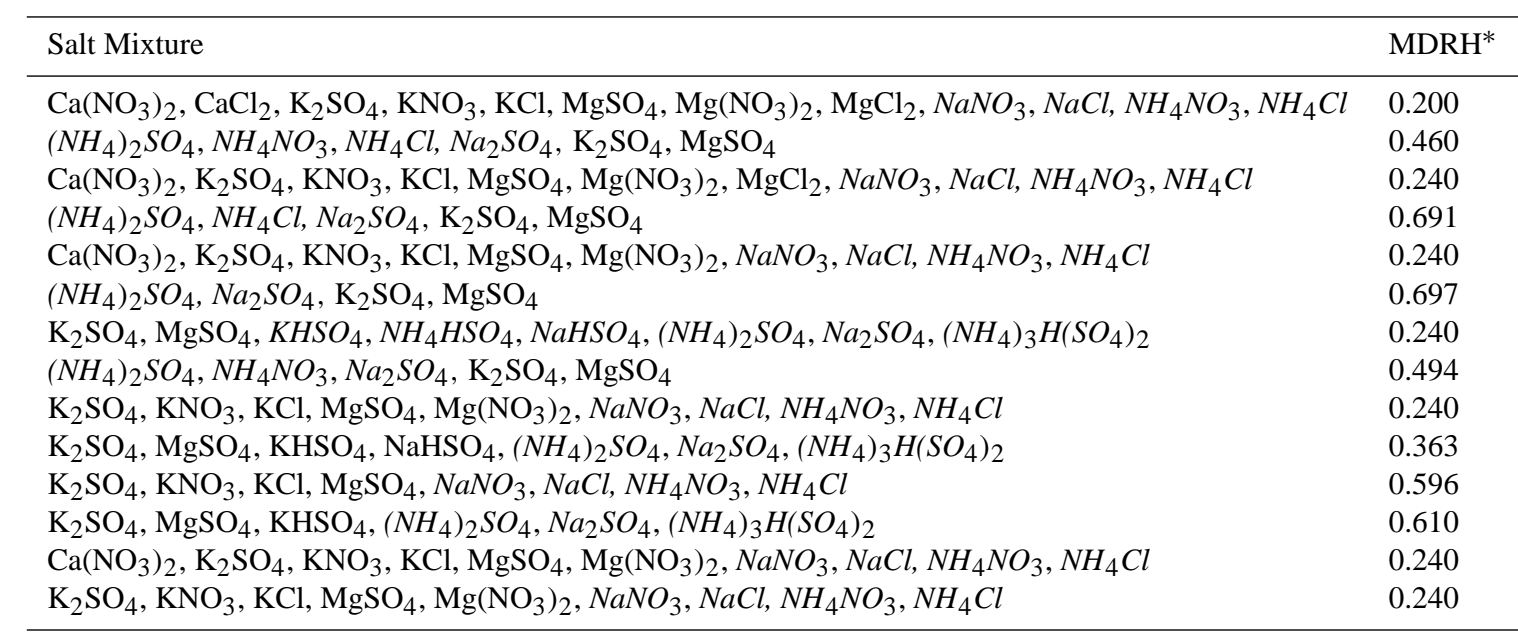

${ }^{1}$ Species in italics determine the mixture for which the MDRH value has been taken.

* Obtained from Potukuchi and Wexler (1995a, b) for mixtures with closest composition (T=298.15 K)

Table 6. Observed and predicted mass fraction of water in the mutual deliquscence region, for an equimolar molar mixture of $\mathrm{NaNO}_{3}$ and $\mathrm{Ca}\left(\mathrm{NO}_{3}\right)_{2}$ at $298.15 \mathrm{~K}$. Data obained from Choi and Chan (2002)

\begin{tabular}{ccc}
\hline $\begin{array}{c}\text { Water mass } \\
\text { fraction (observed) }\end{array}$ & $\begin{array}{c}\text { Water mass } \\
\text { fraction (ISORROPIA II) }\end{array}$ & $a_{w}$ \\
\hline 0.381 & 0.368 & 0.4609 \\
0.373 & 0.363 & 0.4451 \\
0.364 & 0.352 & 0.4258 \\
0.356 & 0.342 & 0.4087 \\
0.342 & 0.336 & 0.3952 \\
0.336 & 0.320 & 0.3743 \\
0.319 & 0.309 & 0.3511 \\
0.299 & 0.291 & 0.3203 \\
0.281 & 0.271 & 0.2841 \\
0.259 & 0.253 & 0.2457 \\
\hline
\end{tabular}

are subsequently dissolved through the equilibria described above. The same is assumed for the dissolved undissociated ammonia, nitric and hydrochloric acid in the aqueous phase $\left(\mathrm{NH}_{3(\mathrm{aq})}, \mathrm{HNO}_{3(\mathrm{aq})}, \mathrm{HCl}_{(\mathrm{aq})}\right)$.

- For sulfate poor cases $\left(\mathrm{R}_{1}>2\right)$ bisulfate ion $\left(\mathrm{HSO}_{4}^{-}\right)$ is considered a minor species from the reaction $\mathrm{HSO}_{4(\mathrm{aq})}^{-} \leftrightarrow \mathrm{H}_{(\mathrm{aq})}^{+}+\mathrm{SO}_{4(\mathrm{aq})}^{2-}$ (see Table 3).

- MDRH points for multicomponent mixtures containing crustal species are not known; they are approximated instead with data for known mixtures with as similar as possible composition (Table 5). For example, the MDRH point for a $\left(\mathrm{NH}_{4}\right)_{2} \mathrm{SO}_{4(\mathrm{~s})}-\mathrm{NH}_{4} \mathrm{NO}_{3(\mathrm{~s})^{-}}$
$\mathrm{NH}_{4} \mathrm{Cl}_{(\mathrm{s})}-\mathrm{Na}_{2} \mathrm{SO}_{4(\mathrm{~s})}-\mathrm{K}_{2} \mathrm{SO}_{4(\mathrm{~s})}-\mathrm{MgSO}_{4}(\mathrm{~s})$ mixture is (not known and) assumed to be the same as for the $\left(\mathrm{NH}_{4}\right)_{2} \mathrm{SO}_{4(\mathrm{~s})}-\mathrm{NH}_{4} \mathrm{NO}_{3(\mathrm{~s})}-\mathrm{NH}_{4} \mathrm{Cl}_{(\mathrm{s})}-\mathrm{Na}_{2} \mathrm{SO}_{4}(\mathrm{~s}) \quad$ mixture. The absence of crustal species in the consideration of the MDRH points of those mixtures is expected to introduce small underprediction of water, since i) both potassium and magnesium have similar deliquescence properties with sodium (Moya et al., 2001a), and, ii) highly insoluble salts (i.e., $\mathrm{CaSO}_{4(\mathrm{~s})}$ ) do not significantly impact water activity, hence do not significantly contribute to DRH depression.

$-\mathrm{OH}_{(\mathrm{aq})}^{-}$is assumed a minor species.

- When crustal species are in excess compared to all the anions, ISORROPIA II assumes that the solution is close to neutral $(\mathrm{pH} \approx 7)$. This is consistent with a presence of excess carbonate in the aerosol phase, which has a $\mathrm{pK}_{a}$ of $\sim 6.4$ (Meng et al., 1995).

- Organic anions are not considered in the thermodynamic calculations, hence their effect on water activity and acidity is neglected. Non-volatile anions (crustal species and sodium) that may be associated with organic acids are treated as "excess", and not considered in ISORROPIA II.

- The DRH of $\mathrm{NH}_{4} \mathrm{NO}_{3 \text { (s) }}$ is strongly dependent on temperature. Under low temperature conditions $(\mathrm{T}<270 \mathrm{~K}$ ), this changes the order (starting from low to high RH) with which salts deliquesce (Fig. 2). For these cases the DRH of $\mathrm{NH}_{4} \mathrm{NO}_{3}$ (s) in ISORROPIA II is assumed to not "cross over" the DRH of the other salts present in the solution, especially 
Table 7. Coefficients of $m\left(a_{w}\right)$ from the polynomial fit $m\left(a_{w}\right)=k_{0}+k_{1} a_{w}+k_{2} a_{w}^{2}+\ldots$

\begin{tabular}{lllllll}
\hline Species & $k_{0}$ & $k_{1}$ & $k_{2}$ & $k_{3}$ & $k_{4}$ & $k_{5}$ \\
\hline $\mathrm{Ca}\left(\mathrm{NO}_{3}\right)_{2}^{\diamond}$ & 36.356 & -165.66 & 447.46 & -673.55 & 510.91 & -155.56 \\
$\mathrm{CaCl}_{2}^{*}$ & 20.847 & -97.599 & 273.220 & -422.120 & 331.160 & -105.450 \\
$\mathrm{CaSO}_{4}$ & $\mathrm{~N} / \mathrm{A}$ & $\mathrm{N} / \mathrm{A}$ & $\mathrm{N} / \mathrm{A}$ & $\mathrm{N} / \mathrm{A}$ & $\mathrm{N} / \mathrm{A}$ & $\mathrm{N} / \mathrm{A}$ \\
$\mathrm{KHSO}_{4}^{\S}$ & 1.061 & -0.101 & $1.579 \times 10^{-2}$ & $-1.950 \times 10^{-3}$ & $9.515 \times 10^{-5}$ & $-1.547 \times 10^{-6}$ \\
$\mathrm{~K}_{2} \mathrm{SO}_{4}^{*}$ & 1061.51 & -4748.97 & 8096.16 & -6166.16 & 1757.47 & 0 \\
$\mathrm{KNO}_{3}^{*}$ & $1.2141 \times 10^{4}$ & $-5.1173 \times 10^{4}$ & $8.1252 \times 10^{4}$ & $-5.7527 \times 10^{4}$ & $1.5305 \times 10^{4}$ & 0 \\
$\mathrm{KCl}^{\star}$ & 179.721 & -721.266 & 1161.03 & -841.479 & 221.943 & 0 \\
$\mathrm{MgSO}_{4}^{\diamond}$ & -0.778 & 177.740 & -719.790 & 1174.600 & -863.440 & 232.310 \\
${\mathrm{Mg}\left(\mathrm{NO}_{3}\right)_{2}^{\diamond}}^{12.166}$ & -16.154 & 0 & 10.886 & 0 & -6.815 \\
$\mathrm{MgCl}_{2}^{\diamond}$ & 11.505 & -26.518 & 34.937 & -19.829 & 0 & 0 \\
\hline
\end{tabular}

- source: Kelly and Wexler (2005)

* source: Kim and Seinfeld (1995)

* source: Kelly and Wexler (2006)

$\S$ Same as $\mathrm{NaHSO}_{4}$

$\diamond$ source: Ha and Chan (1999)

since thermodynamic data for supercooled $\mathrm{NH}_{4} \mathrm{NO}_{3}$ (s) solutions are not known. The same is assumed for $\mathrm{NH}_{4} \mathrm{Cl}_{(\mathrm{s})}$ and $\mathrm{NaNO}_{3(\mathrm{~s})}$ which exhibit similar behavior with $\mathrm{NH}_{4} \mathrm{NO}_{3 \text { (s) }}$ (Fig. 2).

- $\gamma_{\mathrm{OH}^{-}}$and $\gamma_{\mathrm{H}^{+}}$are assumed equal to unity, as the activity coefficient routines cannot explicitly calculate them.

- The temperature dependence of DRH for $\mathrm{Ca}\left(\mathrm{NO}_{3}\right)_{2(\mathrm{~s})}$, $\mathrm{CaCl}_{2(\mathrm{~s})}, \mathrm{Mg}\left(\mathrm{NO}_{3}\right)_{2(\mathrm{~s})}$ and $\mathrm{MgCl}_{2(\mathrm{~s})}$ has been calculated using thermodynamic data for the most hydrated forms of these salts (i.e. $\mathrm{Ca}\left(\mathrm{NO}_{3}\right)_{2} \cdot 4 \mathrm{H}_{2} \mathrm{O}, \mathrm{CaCl}_{2} \cdot 6 \mathrm{H}_{2} \mathrm{O}$, $\mathrm{Mg}\left(\mathrm{NO}_{3}\right)_{2} \cdot 6 \mathrm{H}_{2} \mathrm{O}$ and $\mathrm{MgCl}_{2} \cdot 6 \mathrm{H}_{2} \mathrm{O}$, respectively) as suggested by Kelly and Wexler (2005). DRH values and equilibrium constants of these salts also correspond to their most hydrated forms.

- Double salts (except for $\left.\left(\mathrm{NH}_{4}\right)_{3} \mathrm{H}\left(\mathrm{SO}_{4}\right)_{2(\mathrm{~s})}\right)$ and multiple hydration states are not considered in ISORROPIA II for computational efficiency. While the presence of these species may affect the partitioning of species and the aerosol water content, it is likely that for many atmospherically relevant conditions the effect of these species on model predictions is small (San Martini, 2004; Wexler and Clegg, 2002).

\subsection{ISORROPIA II: new features}

The main improvements to the original ISORROPIA release (Nenes et al., 1998) that are included in ISORROPIA II (and in the latest release of ISORROPIA version 1.7, http://nenes. eas.gatech.edu/ISORROPIA) are:

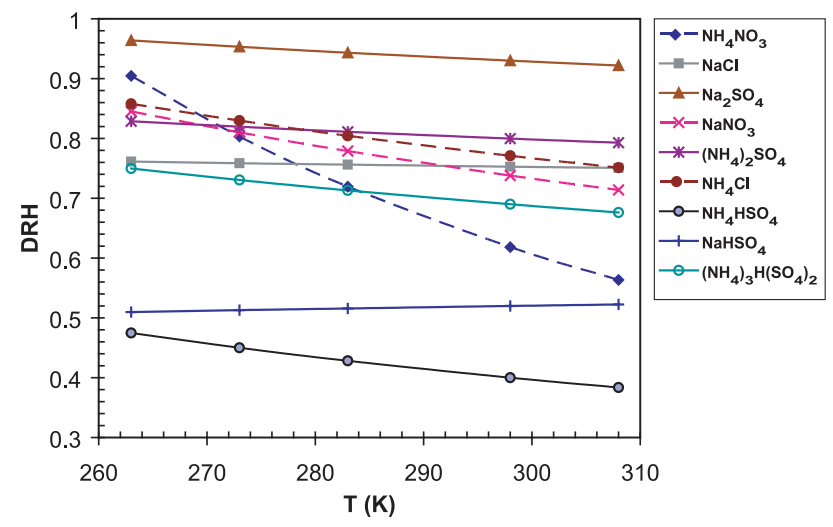

Fig. 2. DRH as a function of temperature for all ISORROPIA salts.

- Gas/liquid/solid partitioning has been extended to include crustal elements that resulted in 10 more salts in the solid phase and 3 more ions in the aqueous phase (Table 3).

- In addition to a thermodynamically stable state the aerosol can also be in a metastable state where no precipitate is formed (always an aqueous solution).

- The water activity database has been updated, using the output from the AIM model (http://www.hpc1.uea.ac. uk/ e770/aim.html).

- Temperature dependency of the activity coefficients is included. This has been done for both pre-calculated tables and online calculations of activity coefficients. 


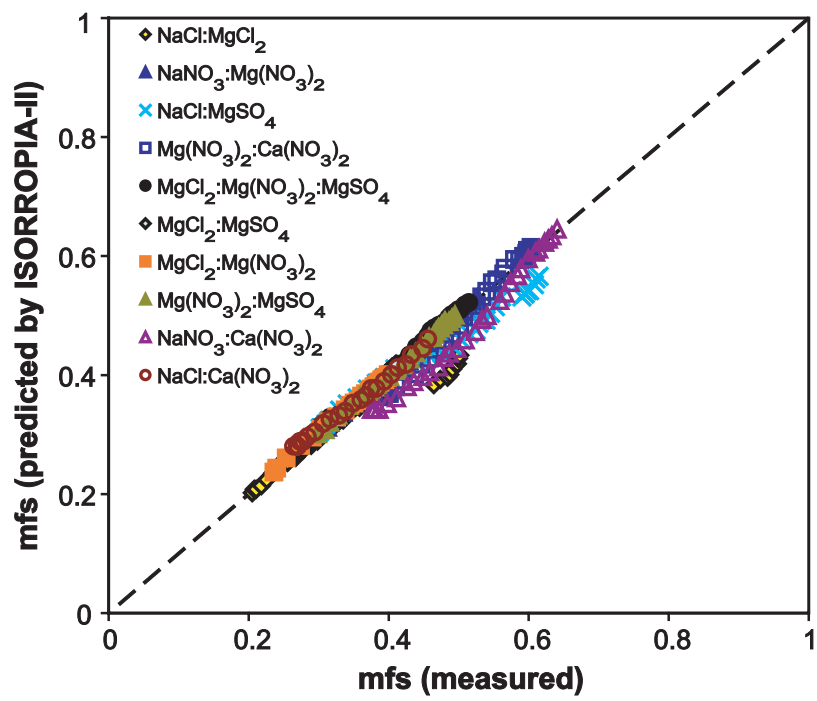

Fig. 3. Comparison of mass fraction of solute as predicted by ISORROPIA II (reverse mode, metastable state) and obtained from experimental measurements (Ha and Chan, 1999; Chan et al., 2000; Choi and Chan, 2002) for a variety of equimolar mixtures of salts. RH ranged between 0.2 and 0.8 for room temperature conditions ( 21 to $\left.23^{\circ} \mathrm{C}\right)$.

- The MDRH points for all the systems considered have been calculated using the GFEMN model of Ansari and Pandis (1999b).

- The activity coefficient calculation algorithm has been optimized to increase computational speed and avoid numerical errors.

- The tabulated Kusik-Meissner binary activity coefficient data have been recomputed through the online calculations for the midpoint of each ionic strength interval.

- A new subroutine has been added to provide the user with the option to "force" ISORROPIA II to conserve mass up to machine precision.

\section{Evaluation of ISORROPIA II}

Salts of calcium, magnesium and sodium with chlorides, nitrates and sulfates are very hygroscopic (Chan et al., 2000). It is useful to assess the capability of ISORROPIA II to predict water activities of various aqueous mixtures of these salts. Water activities $\left(a_{w}\right)$ for such mixtures have been measured with an electrodynamic balance (EDB) from dilute concentrations to high supersaturations (Ha and Chan, 1999; Chan et al., 2000; Choi and Chan, 2002). EDB involves evaporating a levitated droplet through a step decrease of the RH of the surrounding environment. In this way, the mass fraction of as a function of $\mathrm{RH}$ ( or $a_{w}$ ) is obtained. Figure 3 shows comparison of mass fraction of solute as predicted by ISORROPIA II and obtained by experimental data for a variety of equimolar mixtures of salts. The experimental data used here have been taken from Ha and Chan (1999), Chan et al. (2000) and Choi and Chan (2002). RH ranged between 0.20 and 0.80 , while measurements were made at room temperature (21 to $23^{\circ} \mathrm{C}$ ). As seen in Fig. 3, ISORROPIA II predictions agree well with water activity measurements with an average standard deviation (of the difference between predictions and measurements) of \pm 0.018 . A noticeable deviation from the 1:1 line was found for $\mathrm{NaCl}: \mathrm{MgSO}_{4}$ and $\mathrm{NaCl}: \mathrm{MgCl}_{2}$ mixtures under conditions of low RH (due to partial crystallization at low RH, Chan et al., 2000). Other mixtures were found not to crystallize even at RH=30 or 20\% (Ha and Chan, 1999; Chan et al., 2000; Choi and Chan, 2002).

ISORROPIA II is also evaluated against the predictions of SCAPE2 for a wide range of conditions characteristic of urban, remote continental, non-urban continental and marine aerosol (Heitzenberg, 1989; Fitzgerald, 1991; Ansari and Pandis, 1999a). For urban and non-urban continental aerosol, sulfates, nitrates and ammonium are usually dominant inorganic species. Sodium and chloride often compose the majority of the marine particulate matter (usually with some crustal species and sulfates present). This classification is mainly qualitative, as mixing between aerosol types often occurs in the atmosphere.

Table 8 lists the 16 different sets of precursor concentrations that were used in the intercomparison study. Sulfuric acid concentrations range between $1.0-5.7 \mu \mathrm{g} \mathrm{m}^{-3}$ for marine and non-urban continental and $10.0-15.0 \mu \mathrm{g} \mathrm{m}^{-3}$ for urban and remote continental aerosol. For the 16 cases considered, conditions $3,4,15$ and 16 are sulfate-rich $\left(\mathrm{R}_{1}<1\right.$ or $1<\mathrm{R}_{1}<2$ ), conditions $1,2,13$ and 14 represent sulfate near-neutral $\left(\mathrm{R}_{1} \approx 2\right)$ aerosol and cases 5-12 are sulfate-poor $\left(\mathrm{R}_{1}>2\right.$, Table 8$)$. For each set of precursor concentrations, composition at thermodynamic equilibrium was calculated for 11 values of RH ranging from $10-98 \%$; temperature was kept fixed at $298.15 \mathrm{~K}$. Both the thermodynamically stable and metastable state solutions of ISORROPIA II are computed.

For the intercomparison study we calculate the normalized mean error (NME), defined as NME $=\frac{\sum_{i}^{n}\left|I_{i}-S_{i}\right|}{\sum_{i}^{n} S_{i}}$, where $I_{i}$ represents predictions of ISORROPIA II for case $i, S_{i}$ predictions of SCAPE2 and $n$ is the total number of cases considered.

Finally, we compare the CPU time requirements between SCAPE2 and ISORROPIA II, stable and metastable solution of ISORROPIA II, as well as between ISORROPIA II and ISORROPIA for all the simulation conditions of Table 8 . 

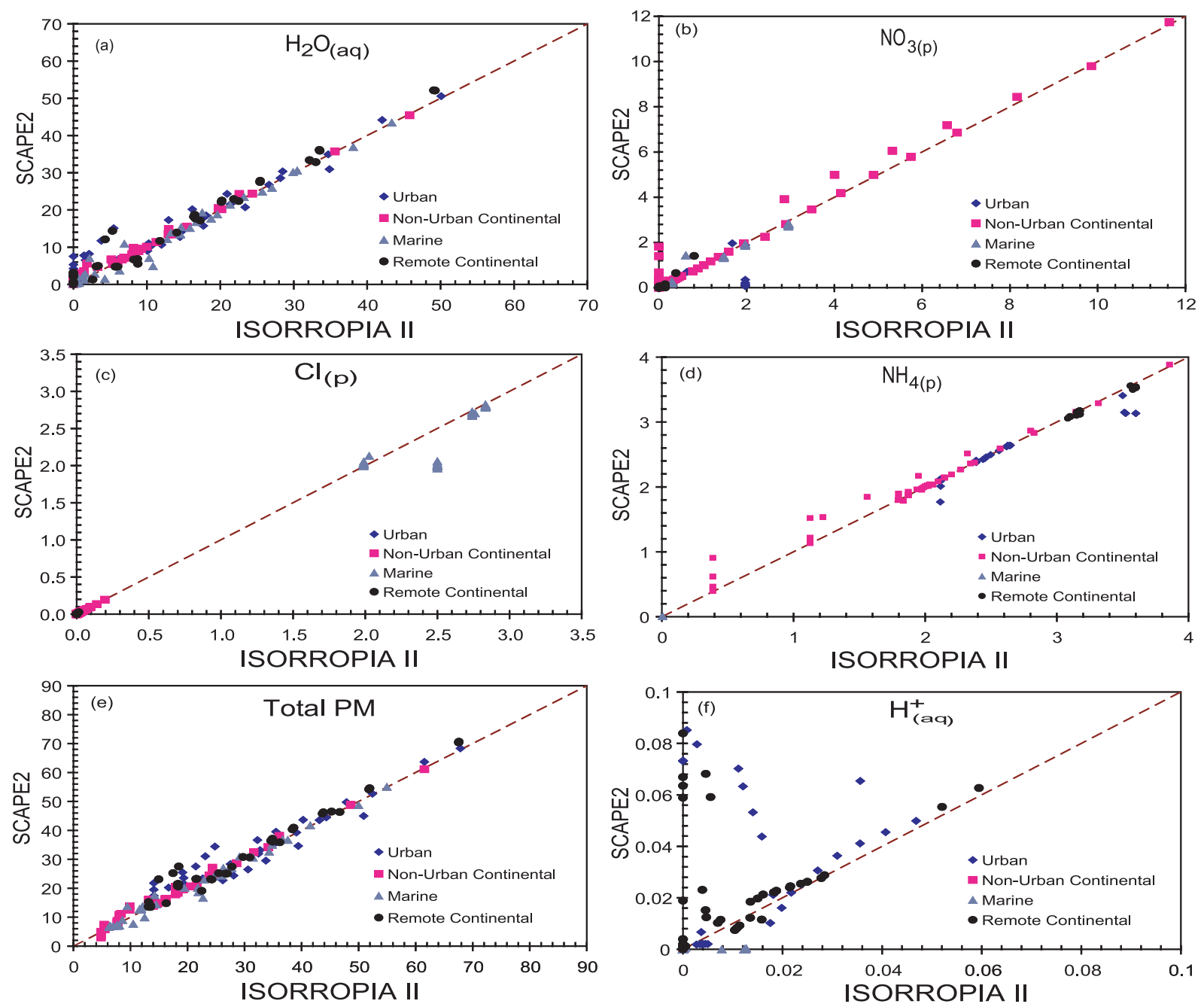

Fig. 4. Concentration of aerosol water (a), nitrate (b), chloride (c), ammonium (d), total PM (e), and hydrogen (f), as predicted by ISORROPIA II (thermodynamically stable solution) and SCAPE2 for all the conditions described in Table 8. Temperature is set to $298.15 \mathrm{~K}$. All units are in $\mu \mathrm{g} \mathrm{m}^{-3}$.

\subsection{Overall assessment of ISORROPIA II vs. SCAPE2}

For all the simulations performed in this work, the water activity database of SCAPE2 was replaced with the one used in ISORROPIA II (reflecting current knowledge on water uptake). Activities of aqueous species in SCAPE2 were computed using Bromley's formula for multicomponent activity coefficients and the Kussik-Meissner method for binary coefficients.

In Fig. 4 we compare predictions of aerosol water, nitrate, chloride, ammonium, total PM and hydrogen concentrations between ISORROPIA II (stable solution, forward problem solved), and SCAPE2 for the conditions specified in Table 8. Both models predict similar amount of aerosol water content (Fig. 4a) with a normalized mean error of $13.5 \%$. Most of this discrepancy is found in the low $\mathrm{RH}$ regimes $(\mathrm{RH}<60 \%)$ where SCAPE2 predicts higher water concentration compared to ISORROPIA II. This discrepancy is attributed to a) non-convergence of SCAPE2, which is corroborated by the large CPU time required for obtaining a solution (see Table 10), and, b) inaccuracies in the activity coefficient model (both binary and multicomponent). At low RH the aqueous phase is highly non-ideal (i.e., low liquid water content, hence high ionic strength), consequently small changes in activity coefficients may result in large changes in the dissolved species concentrations and predicted water uptake. On average, at RH $<60 \%$ ISORROPIA II predicts $I>40$. A few cases exist (for $\mathrm{RH}>65 \%$ ) for which ISORROPIA II predicts less aerosol water than SCAPE2 (Fig. 4a); this originates from differences in aerosol nitrate which then affects water uptake. For a few marine cases, SCAPE2 predicts negligible water due to non-convergence (Fig. 4a). 

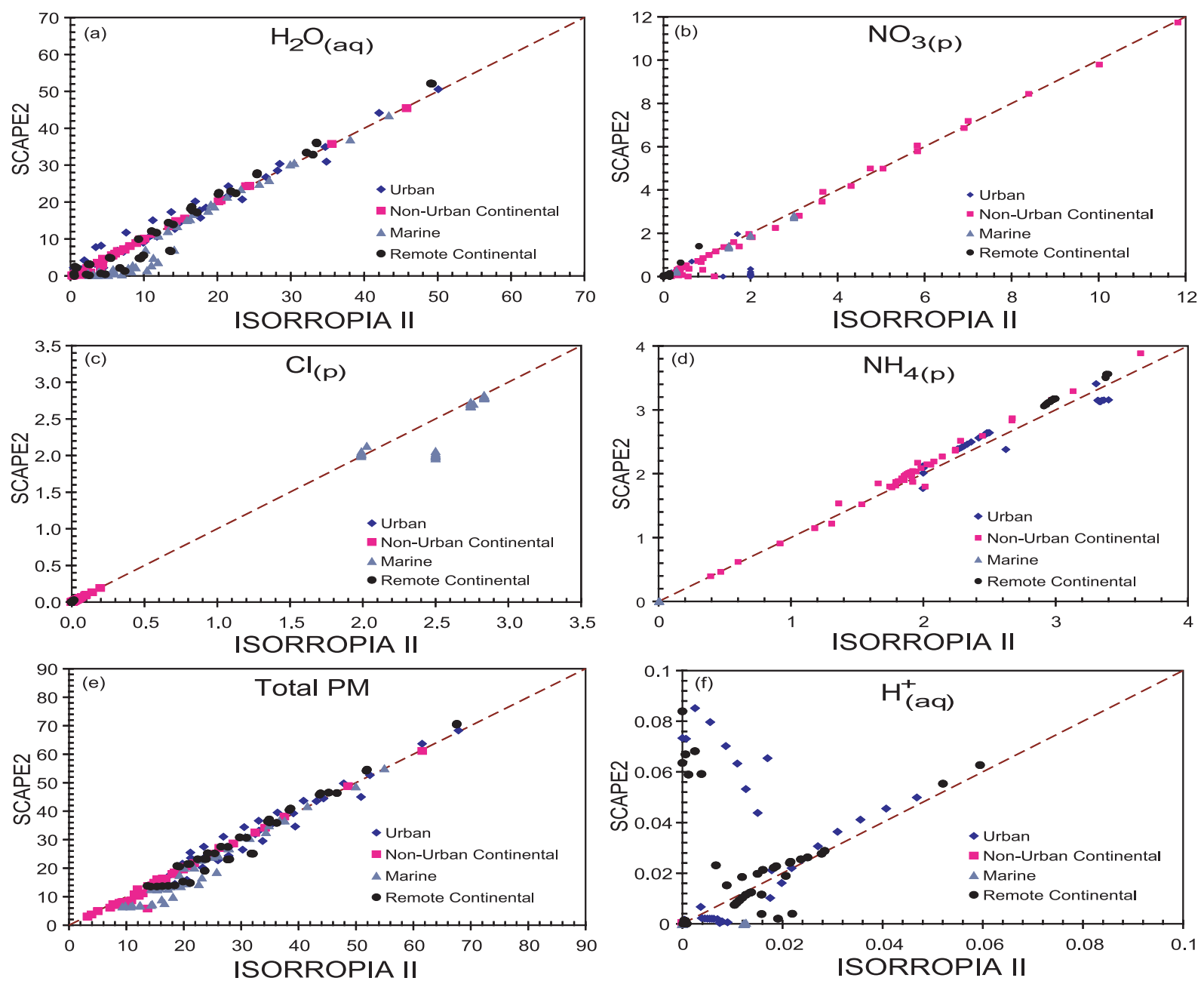

Fig. 5. Same as Fig. 4 but using the metastable solution of ISORROPIA II. All units are in $\mu \mathrm{g} \mathrm{m}^{-3}$.

In Fig. 4b, total aerosol nitrate concentrations are compared for all the input conditions of Table 8. Overall, the agreement is very good with a mean error of $16.5 \%$. ISORROPIA II predicts non-negligible amount of nitrate for some urban cases while SCAPE2 does not. For a few non-urban continental cases, ISORROPIA II underpredicts aerosol nitrate compared to SCAPE2. The sources of these discrepancies are further investigated through specific examples in Sect. 4.2.

Aerosol chloride concentration predictions are shown in Fig. 4c where both models show similar results $(\mathrm{NME}=6.5 \%)$; small discrepancies are seen for a few marine cases (due to non-convergence of SCAPE2 solution) in which chloride exists in significant amount.

For aerosol ammonium predictions (Fig. 4d), no substantial differences between the two models were found $(\mathrm{NME}=2.1 \%)$. Discrepancies were primarily found in some non-urban continental cases that represent a sulfate-poor, ammonium-rich environment and are further analyzed in
Sect. 4.2. Even though a few differences exist in the predicted concentrations of semi-volatile species, the total PM composition (Fig. 4e) shows very good agreement (NME $=13.0 \%)$. The worst agreement between the two models was seen for $\mathrm{H}^{+}$predictions (Fig. 4f) with the normalized mean error significantly higher than for any other component $(\mathrm{NME}=64 \%)$. The discrepancy occurs at low RH (as it scales with water content).

SCAPE2 predictions are also compared against the metastable state solution of ISORROPIA II (Fig. 5). Table 9 shows normalized mean errors between ISORROPIA II (both stable and metastable solutions) and SCAPE2 for the simulations of Table 8. As can be seen in Figs. 4, 5 and Table 9, the stable state predictions of ISORROPIA II are closer to SCAPE2 predictions. This is expected since only for low RHs $(<40 \%)$ SCAPE2 solution, by always attempting to solve for a liquid phase, deviates from the stable state behavior, moving towards the metastable state (see Sect. 3.2). 
Table 8. List of input conditions for model simulations ${ }^{\mathrm{a}}$.

\begin{tabular}{cllllllllll}
\hline Case & Aerosol Type & $\mathrm{Na}$ & $\mathrm{H}_{2} \mathrm{SO}_{4}$ & $\mathrm{NH}_{3}$ & $\mathrm{HNO}_{3}$ & $\mathrm{HCl}$ & $\mathrm{Ca}^{2+}$ & $\mathrm{K}^{+}$ & $\mathrm{Mg}^{2+}$ & $\mathrm{R}_{1}, \mathrm{R}_{2}, \mathrm{R}_{3}$ \\
\hline 1 & Urban (1) & 0.000 & 10.000 & 3.400 & 2.000 & 0.000 & 0.400 & 0.330 & 0.000 & $2.14,0.18,0.18$ \\
2 & Urban (2) & 0.023 & 10.000 & 3.400 & 2.000 & 0.037 & 0.900 & 1.000 & 0.000 & $2.44,0.48,0.47$ \\
3 & Urban (3) & 0.000 & 15.000 & 2.000 & 10.000 & 0.000 & 0.900 & 1.000 & 0.000 & $1.27,0.31,0.32$ \\
4 & Urban (4) & 0.000 & 15.000 & 2.000 & 10.000 & 0.000 & 0.400 & 0.330 & 0.000 & $0.89,0.12,0.12$ \\
5 & N-u Cont. (1) & 0.200 & 2.000 & 8.000 & 12.000 & 0.200 & 0.120 & 0.180 & 0.000 & $23.9,0.80,0.37$ \\
6 & N-u Cont. (2) & 0.100 & 4.000 & 10.000 & 7.000 & 0.100 & 0.120 & 0.180 & 0.050 & $14.8,0.34,0.24$ \\
7 & N-u Cont. (3) & 0.023 & 5.664 & 12.000 & 2.000 & 0.037 & 0.120 & 0.180 & 0.050 & $12.4,0.18,0.17$ \\
8 & N-u Cont. (4) & 0.023 & 5.664 & 20.400 & 0.611 & 0.037 & 0.120 & 0.180 & 0.000 & $20.9,0.15,0.13$ \\
9 & Marine (1) & 2.000 & 1.000 & 0.010 & 0.300 & 3.121 & 0.100 & 0.100 & 0.070 & $9.36,9.30,0.80$ \\
10 & Marine (2) & 1.500 & 1.000 & 0.010 & 1.500 & 2.500 & 0.360 & 0.450 & 0.050 & $8.66,8.60,2.21$ \\
11 & Marine (3) & 2.500 & 3.000 & 0.001 & 3.000 & 2.500 & 0.500 & 1.000 & 0.050 & $4.86,4.86,1.31$ \\
12 & Marine (4) & 3.000 & 3.000 & 0.020 & 2.000 & 3.121 & 0.360 & 0.450 & 0.130 & $5.14,5.10,0.84$ \\
13 & Rem. Cont. (1) & 0.000 & 10.000 & 4.250 & 0.145 & 0.000 & 0.080 & 0.090 & 0.000 & $2.49,0.04,0.04$ \\
14 & Rem. Cont. (2) & 0.023 & 10.000 & 3.000 & 1.000 & 0.037 & 0.080 & 0.090 & 0.000 & $1.78,0.05,0.04$ \\
15 & Rem. Cont. (3) & 0.100 & 15.000 & 3.000 & 4.000 & 0.100 & 0.080 & 0.090 & 0.000 & $1.21,0.06,0.03$ \\
16 & Rem. Cont. (4) & 0.200 & 15.000 & 3.000 & 8.000 & 0.200 & 0.080 & 0.090 & 0.040 & $1.25,0.10,0.04$ \\
\hline
\end{tabular}

a Simulations for each case were conducted for $10,25,40,55,65,70,75,80,85,90$ and $98 \%$ relative humidity. Temperature was set to 298.15 K. Concentration given in $\mu \mathrm{g} \mathrm{m}^{-3}$.

b N-u Cont., non-urban continental; Rem. Cont., remote continental.
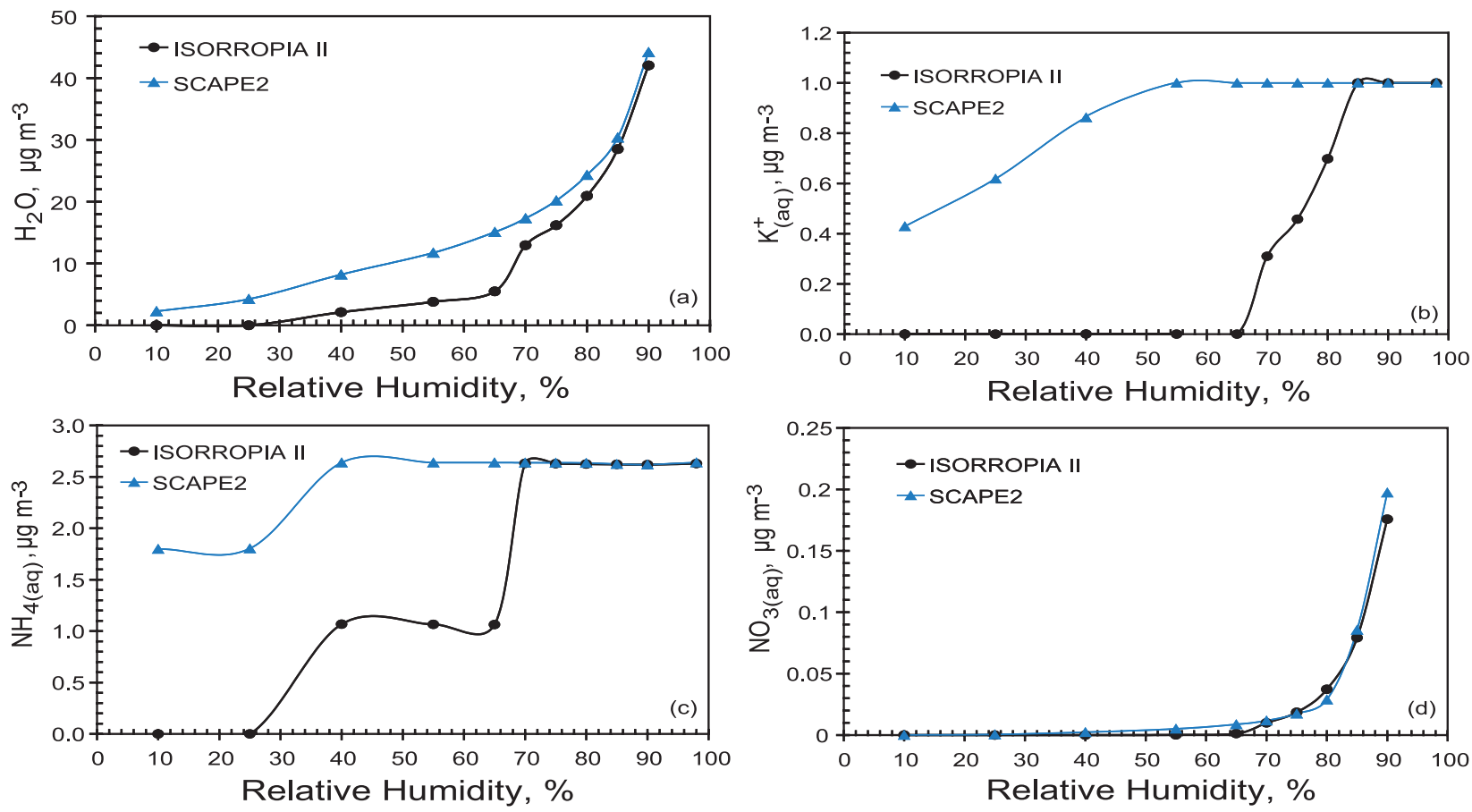

Fig. 6. Concentration of aerosol water (a), aqueous potassium (b), aqueous ammonium (c), and aqueous nitrate (d) as predicted by ISORROPIA II (thermodynamically stable solution) and SCAPE2 for the urban (1) case (Table 8) corresponding to a sulfate rich aerosol behavior $\left(1<R_{1}<2\right)$. Temperature is set to $298.15 \mathrm{~K}$. 

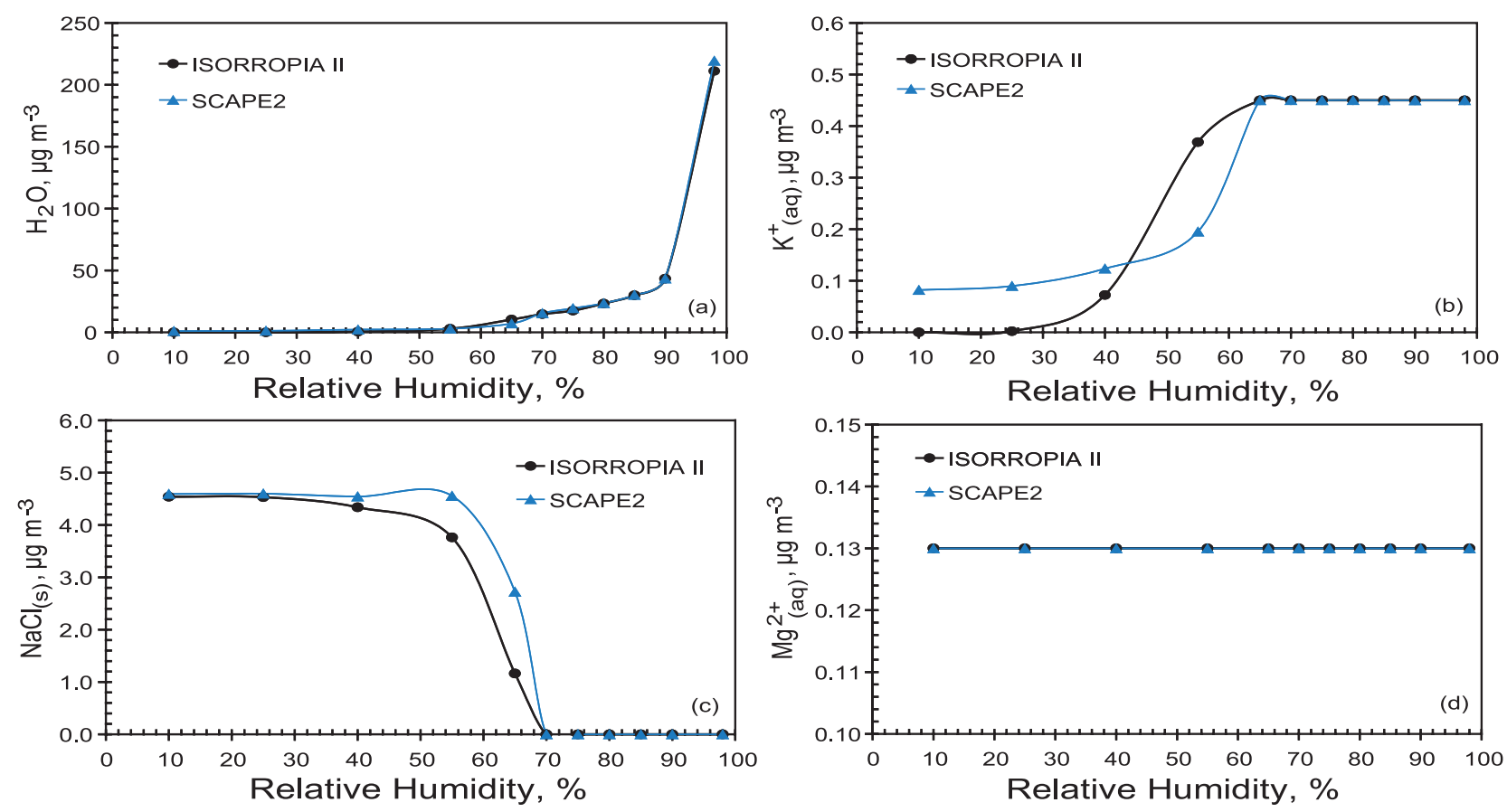

Fig. 7. Concentration of aerosol water (a), aqueous potassium (b), solid sodium chloride (c), and aqueous magnesium (d) as predicted by ISORROPIA II (thermodynamically stable solution) and SCAPE2 for the marine (4) case (Table 8) corresponding to a sulfate poor, crustal and sodium rich aerosol behavior $\left(R_{1}>2, R_{2}>2\right)$. Temperature is set to $298.15 \mathrm{~K}$.

Table 9. Normalized mean errors between ISORROPIA II and SCAPE2 for the simulations in Table 8.

\begin{tabular}{lllllll}
\hline NME (\%) & $\mathrm{H}_{2} \mathrm{O}_{(p)}$ & $\mathrm{NO}_{3(p)}$ & $\mathrm{Cl}_{(p)}$ & $\mathrm{NH}_{4(p)}$ & Total PM & $\mathrm{H}_{(\mathrm{aq})}^{+}$ \\
\hline ISORROPIA II (Stable) & 13.5 & 16.5 & 6.5 & 2.1 & 13.0 & 64.9 \\
ISORROPIA II (Metastable) & 14.7 & 23.7 & 6.6 & 6.7 & 14.3 & 68.0 \\
\hline
\end{tabular}

\subsection{Understanding the discrepancies between ISOR- ROPIA II and SCAPE2}

The previous discussion provides an overall intercomparison of the two models for a broad RH and composition domain; some cases are further examined to understand the causes of discrepancies. In Fig. 6 we compare aerosol water content, aqueous phase potassium, aqueous phase ammonium and aqueous phase nitrate concentration predictions for case 3 (see Table 8) which produced the largest discrepancy in aerosol water and total PM concentrations. This case represents an urban type aerosol with the solution being highly acidic $\left(R_{1}=1.27\right)$. Under such conditions, the water content discrepancy between the models is largest for low RHs for the reasons outlined in Sect. 3.2 (i.e., differences in solution dynamics). This is clearly shown in Fig. 5a where only for RH $>70 \%$ SCAPE2 and ISORROPIA II closely follow each other. SCAPE2 predicts significant amount of aqueous phase potassium (Fig. 6b) and ammonium (Fig. 6c) at low relative humidities, while ISORROPIA II predicts gradual deliquescence of $\mathrm{K}_{2} \mathrm{SO}_{4}$ from $65 \%$ to $85 \% \mathrm{RH}$. However, SCAPE2 predicts complete deliquescence of $\mathrm{K}_{2} \mathrm{SO}_{4}$ at $\mathrm{RH}=55 \%$ which may be due to non-convergence of its numerical solution. Particulate phase ammonium is mainly present as ammonium bisulfate $\left(\mathrm{NH}_{4} \mathrm{HSO}_{4(s)}\right)$ and letovicite $\left(\left(\mathrm{NH}_{4}\right)_{3} \mathrm{H}\left(\mathrm{SO}_{4}\right)_{2(s)}\right)$ in ISORROPIA II. However, SCAPE2 predicts the formation of $\left(\mathrm{NH}_{4}\right)_{3} \mathrm{H}\left(\mathrm{SO}_{4}\right)_{2}$ only, throughout the whole RH regime. This can also be seen in Fig. 6c where ISORROPIA II predicts a two-step dissolution of ammonium; one at $\mathrm{RH}=40 \%$ from the deliquescence of $\left(\mathrm{NH}_{4}\right)_{3} \mathrm{H}\left(\mathrm{SO}_{4}\right)_{2(\mathrm{~s})}$ and one at $\mathrm{RH}=70 \%$ from the deliquescence of $\mathrm{NH}_{4} \mathrm{HSO}_{4}$ (s). Water uptake with SCAPE2 exhibits deliquescence only of $\left(\mathrm{NH}_{4}\right)_{3} \mathrm{H}\left(\mathrm{SO}_{4}\right)_{2}$ (s) at $\mathrm{RH}=40 \%$. Both models predict similar amounts of aqueous phase nitrate for all RHs (Fig. 6d) which justifies the assumption of ISORROPIA II for considering $\mathrm{NO}_{3(\mathrm{aq})}^{-}$a minor species for sulfate-rich cases. 
Table 10. CPU time required for the simulations in Table 8.

\begin{tabular}{|c|c|c|c|c|c|c|}
\hline Aerosol case & $\begin{array}{l}\text { Convergence } \\
\text { criterion }\end{array}$ & $\begin{array}{l}\text { ISORROPIA II } \\
\text { (stable) } \\
\text { CPU time }(\mathrm{ms})\end{array}$ & $\begin{array}{l}\text { SCAPE2 } \\
\text { CPU time (ms) }\end{array}$ & $\frac{\text { CPU }_{\text {SCAPE2 }}}{\text { CPU IISORROPIAII }^{-}}$ & $\frac{\text { CPU ISORROPIAII(stable) }_{\text {CPU ISORROPIAII(metastable) }}}{\text { CPST }_{\text {IIf }}}$ & $\frac{\text { CPU }_{\text {ISORROPIAII }}}{\text { CPU ISORROPIA }^{\text {ISOROA }}}$ \\
\hline $\begin{array}{l}\text { Marine } \\
\text { Urban } \\
\text { Remote continental } \\
\text { Non-urban continental }\end{array}$ & $10^{-3}$ & $\begin{array}{l}30 \\
20 \\
20 \\
20\end{array}$ & $\begin{array}{l}50 \\
210 \\
440 \\
110\end{array}$ & $\begin{array}{l}16.7 \\
10.5 \\
22.0 \\
5.5\end{array}$ & $\begin{array}{l}1.16 \\
1.09 \\
1.05 \\
1.00\end{array}$ & $\begin{array}{l}1.0 \\
1.0 \\
1.0 \\
1.0\end{array}$ \\
\hline $\begin{array}{l}\text { Marine } \\
\text { Urban } \\
\text { Remote continental } \\
\text { Non-urban continental }\end{array}$ & $10^{-4}$ & $\begin{array}{l}30 \\
20 \\
20 \\
20\end{array}$ & $\begin{array}{l}>1000 \\
280 \\
>1000 \\
420\end{array}$ & $\begin{array}{l}>1000 \\
14.0 \\
>1000 \\
21.0\end{array}$ & $\begin{array}{l}1.16 \\
1.09 \\
1.05 \\
1.00\end{array}$ & $\begin{array}{l}1.0 \\
1.0 \\
1.0 \\
1.0\end{array}$ \\
\hline $\begin{array}{l}\text { Marine } \\
\text { Urban } \\
\text { Remote continental } \\
\text { Non-urban continental }\end{array}$ & $10^{-5}$ & $\begin{array}{l}30 \\
20 \\
20 \\
20\end{array}$ & $\begin{array}{l}>1000 \\
>1000 \\
>1000 \\
1250\end{array}$ & $\begin{array}{l}>1000 \\
>1000 \\
>1000 \\
62.50\end{array}$ & $\begin{array}{l}1.16 \\
1.09 \\
1.05 \\
1.00\end{array}$ & $\begin{array}{l}1.0 \\
1.0 \\
1.0 \\
1.0\end{array}$ \\
\hline
\end{tabular}

Figure 7 shows comparison of aerosol water, $\mathrm{NaCl}_{(\mathrm{s})}$ and $\mathrm{K}_{(\mathrm{aq})}$ and $\mathrm{Mg}_{(\mathrm{aq})}$ predictions for case 12 (see Table 8), which is a sulfate poor, sodium and crustal species rich aerosol $\left(R_{1}=5.1\right)$. The two models agree well (mean error of 5.1\%) in aerosol water content predictions (Fig. 6a). SCAPE2, however, predicts significantly higher aqueous potassium for $\mathrm{RH}<40 \%$ (Fig. 6b). This is mainly due to different approaches used to associate $\mathrm{K}_{(\mathrm{aq})}$ with $\mathrm{NO}_{3(\mathrm{aq})}$ and $\mathrm{SO}_{4(\mathrm{aq})}$ at low RH. ISORROPIA II uses the principle of "compositional invariance" (Sect. 3.2), hence it preferentially associates $\mathrm{K}_{(\mathrm{aq})}$ with $\mathrm{SO}_{4(\mathrm{aq})}$ to form $\mathrm{K}_{2} \mathrm{SO}_{4(\mathrm{~s})}$, and then $\mathrm{KNO}_{3(\mathrm{~s})}$ and $\mathrm{KCl}_{(\mathrm{s})}$. SCAPE2 tends to associate $\mathrm{K}_{(\mathrm{aq})}$ with $\mathrm{NO}_{3(\mathrm{aq})}$ as $\mathrm{KNO}_{3(\mathrm{~s})}$, then with $\mathrm{Cl}_{(\mathrm{aq})}$ as $\mathrm{KCl}_{(\mathrm{s})}$ and then with $\mathrm{SO}_{4(\mathrm{aq})}$ as $\mathrm{K}_{2} \mathrm{SO}_{4(\mathrm{~s})}$. Since the DRH of $\mathrm{KCl}_{(\mathrm{s})}$ is lower than $\mathrm{K}_{2} \mathrm{SO}_{4(\mathrm{~s})}$, SCAPE2 deliquesces aerosol potassium at a lower RH than ISORROPIA II. Unlike potassium, both models predict the association of sodium between nitrate $\left(\mathrm{NaNO}_{3(\mathrm{~s})}\right)$ and chloride $\left(\mathrm{NaCl}_{(\mathrm{s})}\right)$ in a similar way. This is shown in Fig. 6c where the dissociation of $\mathrm{NaCl}_{(\mathrm{s})}$ as a function of $\mathrm{RH}$ is similar between both models (NME $=12.1 \%$ ). Aqueous magnesium is the same in both models (Fig. 7d), which verifies the ISORROPIA II assumption that $\mathrm{MgSO}_{4(\mathrm{~s})}$ never precipitates out of solution (Sect. 3.3).

In Fig. 8 we compare aerosol water, $\mathrm{NO}_{3(\mathrm{aq})}$ and $\mathrm{NH}_{4(\mathrm{aq})}$ as a function of $\mathrm{RH}$ for case 5 (a sulfate poor, ammonium rich aerosol, $\mathrm{R}_{1}=23.9, \mathrm{R}_{2}=0.80, \mathrm{R}_{3}=0.37$ ). Compared to SCAPE2, ISORROPIA II slightly underpredicts aerosol water, aqueous nitrate and ammonium. This difference is seen between 25 and $65 \% \mathrm{RH}$, because SCAPE2 predicts total deliquescence of sulfates at $\mathrm{RH}=40 \%$ while ISORROPIA II does at $\mathrm{RH}=70 \%$. The increase of water content shifts the equilibrium of $\mathrm{HNO}_{3(\mathrm{~g})} \longleftrightarrow \mathrm{H}_{(\mathrm{aq})}^{+}+\mathrm{NO}_{3(\mathrm{aq})}^{-}$and $\mathrm{NH}_{3(\mathrm{~g})}+\mathrm{H}_{2} \mathrm{O}_{(\mathrm{aq})} \leftrightarrow \mathrm{NH}_{4(\mathrm{aq})}^{+}+\mathrm{OH}_{(\mathrm{aq})}^{-}$to the right predicting more aqueous nitrate (Fig. $7 \mathrm{~b}$ ) and ammonium (Fig. 6c) for the same RH regime.

\subsection{Metastable vs. stable solutions}

The differences between metastable and stable thermodynamic solutions of ISORROPIA II are illustrated in Fig. 9, which shows aerosol water and aqueous potassium concentration as a function of relative humidity for a sulfate nearneutral aerosol (case 13 of Table 8). SCAPE2 is also included for comparison. The thermodynamically stable solution of ISORROPIA II predicts deliquescence of the aerosol mixture at $60 \% \mathrm{RH}$ (DRH of ammonium nitrate). The MDRH for this specific aerosol mixture is 0.46 , which explains the presence of aqueous potassium (and aerosol water) in the deliquescence solution of ISORROPIA II for RH below $60 \%$. As expected, the metastable solution predicts significant amounts of water below the MDRH (and by definition particulate potassium is deliquesced at all RHs). The ionic strength are similar in both metastable and stable solutions (which for RH between 55 and $75 \%$ ranges between 40 and 20), suggesting that the variations in Fig. 9 are from differences in water uptake. SCAPE2 yields a solution that is between the stable and metastable ISORROPIA II. Below 40\% RH, the predicted concentration of aerosol water by SCAPE2 is slightly larger than the stable solution of ISORROPIA II (Fig. 9a). This results in partial dissolution of aerosol potassium $(\mathrm{RH}<40 \%)$ predicted by SCAPE2 as opposed to the stable solution of ISORROPIA II which does not predict deliquescence of aerosol potassium for this RH regime (Fig. 8b). A significant difference in predicted $I$ is also found between ISORROPIA II and SCAPE (e.g., at RH=40\% SCAPE2 predicts $I=85$ while ISORROPIA II (metastable) predicts $I=64$ ).

\section{4 "Forward" vs. "Reverse" problem solution}

ISORROPIA II is designed to solve both forward and reverse problems. It is useful to assess whether the two solution modes predict identical outputs for the same input. For this assessment, the output from the forward problem 

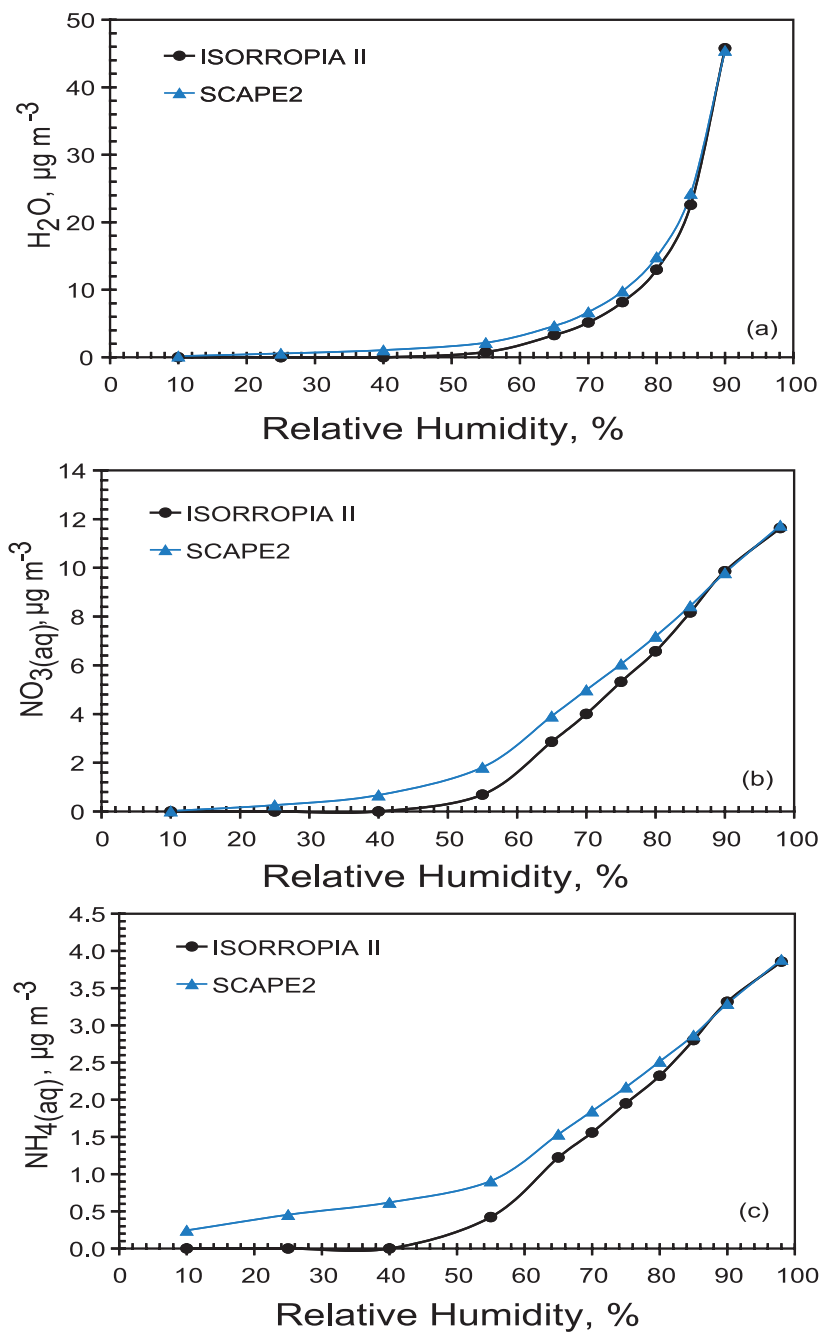

Fig. 8. Concentration of aerosol water (a), aqueous nitrate (b), and aqueous ammonium (c) as a function of relative humidity as predicted by ISORROPIA II (thermodynamically stable solution) and SCAPE2 for the non-urban continental (1) case (Table 8) corresponding to a sulfate poor, ammonium rich aerosol behavior $\left(R_{1}>2\right.$, $R_{2}<2$ ). Temperature is set to $298.15 \mathrm{~K}$.

(particulate phase concentrations of $\mathrm{NH}_{4}, \mathrm{SO}_{4}, \mathrm{Na}, \mathrm{Cl}, \mathrm{NO}_{3}$, $\mathrm{Ca}, \mathrm{K}$, and $\mathrm{Mg}$ ) has been used as input to the reverse problem. The two solution modes are assessed by comparing predictions of aqueous nitrate and sulfate. Since sulfate is only found in the aerosol phase, aqueous phase sulfate calculations are used to evaluate the solid/liquid partitioning behavior between the two solution algorithms, while aqueous nitrate is used as a proxy for gas-aerosol partitioning (for all the conditions specified in Table 8). The agreement between the two solutions was found to be excellent with the NME being $3.4 \pm 1.1 \%$ for aqueous sulfate and $2.5 \pm 1.3 \%$ for aqueous nitrate concentration.
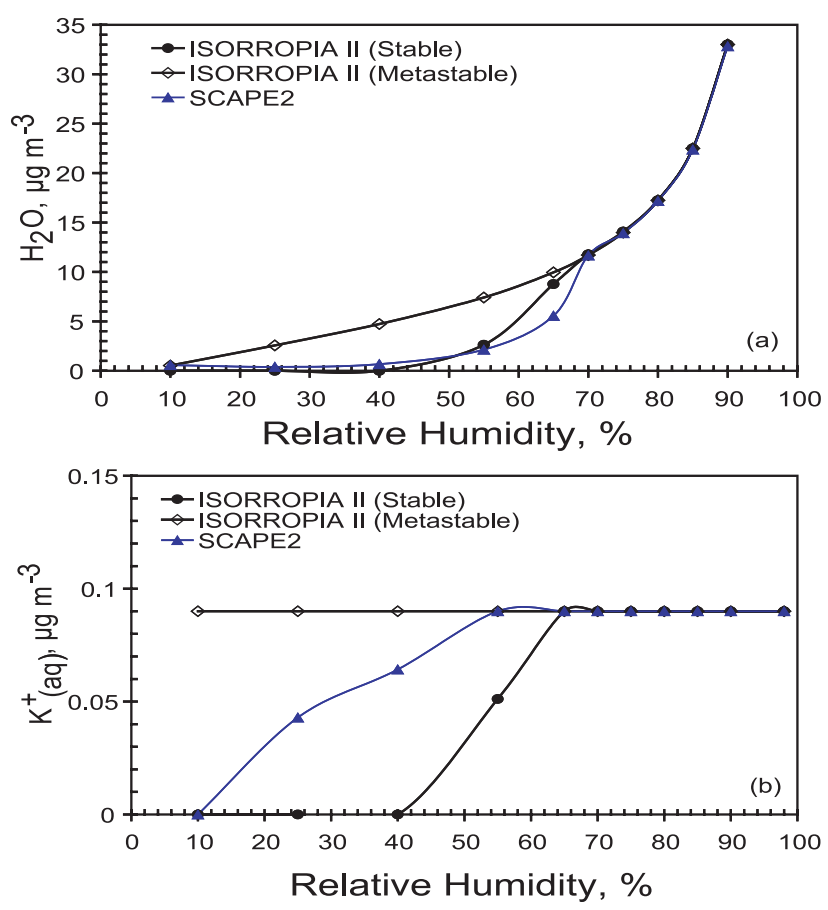

Fig. 9. Concentration of aerosol water (a), and aqueous phase potassium (b) as a function of relative humidity as predicted by ISORROPIA II (using the thermodynamically stable and metastable solutions) and SCAPE2 for the remote continental (1) case (Table 8) corresponding to a sulfate near-neutral aerosol behavior. Temperature is set to $298.15 \mathrm{~K}$.

\subsection{Computational speed}

The timing tests were performed on a Dell 8300 Intel Pentium $4 \mathrm{CPU} 3.20 \mathrm{GHz}, 512 \mathrm{MB}$ of RAM workstation running Windows XP operating system. Both codes were compiled with Watcom FORTRAN compiler version 2.0 with full optimization options on. Table 10 shows the CPU time needed by the two models for the aerosol types described in Table 8 . ISORROPIA II consumes much less CPU time compared to SCAPE2 with the difference being at least an order of magnitude for all aerosol cases. The amount of time required by ISORROPIA II for each aerosol case was found to be approximately the same even if the convergence criterion for solids and water was decreased down to $10^{-6}$ or $10^{-7}$ which is a proof of the rapid and robust convergence of the code. However, larger convergence criterion was used for the intercomparison study (see Table 10), to assure a quick and convergent solution from SCAPE2. For completeness we also compare the CPU time required by ISORROPIA (version 1.7, 15 March 2006) for all the simulation conditions of Table 8, but with crustal species set to zero). Although ISORROPIA II solves for more species than ISORROPIA, it is not slower because of optimizations in the activity coefficient calculation algorithm in ISORROPIA II. Finally, in Table 10 we compare the CPU time required by the stable 
and metastable solutions of ISORROPIA II. As expected, the metastable solution is slightly faster than the stable solution since the absence of solid species requires the solution of fewer equations.

\section{Summary}

A new model, ISORROPIA II, is developed that treats the thermodynamics of $\mathrm{K}^{+}-\mathrm{Ca}^{2+}-\mathrm{Mg}^{2+}-\mathrm{NH}_{4}^{+}-\mathrm{Na}^{+}-\mathrm{SO}_{4}^{2-}-$ $\mathrm{NO}_{3}^{-}-\mathrm{Cl}^{-}-\mathrm{H}_{2} \mathrm{O}$ aerosol systems. A comprehensive evaluation was conducted against the thermodynamic model SCAPE2 (in terms of composition predicted and computational speed) for a wide variety of aerosol conditions that cover typical urban, remote continental, marine and nonurban continental environments. Water uptake predictions are also evaluated against published laboratory experiments for multicomponent mixtures. The overall predictions of aerosol water, total PM and concentration of semi-volatile species were generally comparable between the two models under most conditions. For aerosol water content and total PM mass, the two models agreed within approximately $13 \%$. The normalized mean error for total aerosol nitrate predictions was $16 \%$, while for aerosol chloride and ammonium concentration the agreement was within 2-6\%. Small discrepancies were found to exist between the two models under certain conditions, primarily for relative humidities between 40 and $70 \%$. These discrepancies are mainly attributed to the solution dynamics treatment of water uptake in mutual deliquescence regions and the association of non-volatile cations with sulfate, nitrate and chloride. For all cases examined, ISORROPIA II is more than an order of magnitude faster than SCAPE2.

Future work will focus on evaluating various aspects of ISORROPIA-II, which include i) code performance the transition points across solution subdomains, ii) assessing the impact of using a simplified phase diagram (lack of multiple hydration states and double salts) on thermodynamic predictions, and, iii) evaluation with in-situ data, and within a 3-D modeling framework. Based on the initial comparison with SCAPE2, we can say that ISORROPIA II exhibits robust and rapid convergence, making it one of the most computationally efficient and comprehensive inorganic thermodynamic equilibrium modules available.

Acknowledgements. This work was supported by the National Oceanic and Atmospheric Administration under contract NMRAC000-5-04017. We would like to acknowledge the contributions and support of the whole community that lead to significant improvements of the original ISORROPIA code. In particular we would like to acknowledge the contributions of (in alphabetical order): A. Ansari, V. Bouchet, P. Bhave, S. Clegg, B. Hutzel, K. Capaldo, B. Koo, S. Kreidenweis, P. Makar, F. San Martini, D. Mauzerall, C. Nolte, B. Pun, A. Russell, U. Shankar, D. Tong, J. West, D. Waldron, A. Zakey, and Y. Zhang. We also thank C. Pilinis for his comments on the manuscript.
Edited by: J.-L. Jimenez

\section{References}

Altshüller, A. P.: Atmospheric particle sulfur and sulfur dioxide relationships at urban and nonurban locations, Atmos. Environ., 18, 1421-1431, 1984.

Amundson, N. R., Caboussat, A., He, J. W., Martynenko, A. V., Savarin, V. B., Seinfeld, J. H., and Yoo, K. Y.: A new inorganic atmospheric aerosol phase equilibrium model (UHAERO), Atmos. Chem. Phys., 6, 975-992, 2006,

http://www.atmos-chem-phys.net/6/975/2006/.

Ansari, A. S. and Pandis, S. N.: Prediction of multicomponent inorganic atmospheric aerosol behavior, Atmos. Environ., 33, 745757, 1999a.

Ansari, A. S. and Pandis, S. N.: An analysis of four models predicting the partitioning of semivolatile inorganic aerosol components, Aerosol Sci. Technol., 31, 129-153, 1999 b.

Brauer, M. and Brook, J. R.: Ozone personal exposures and health effects for selected groups residing in the Fraser Valley, Atmos. Environ., 31, 2113-2122, 1997.

Bromley, L. A.: Thermodynamic properties of strong electrolytes in aqueous solutions, AIChE J., 19, 313-320, 1973.

Chan, M. N. and Chan, C. K.: Mass transfer effects in hygroscopic measurements of aerosol particles, Atmos. Chem. Phys., 5, 2703-2712, 2005,

http://www.atmos-chem-phys.net/5/2703/2005/.

Clegg, S. L. and Pitzer, K. S.: Thermodynamics of multicomponent, miscible, ionic solutions: generalized equations for symmetrical electrolytes, J. Phys. Chem., 96, 3513-3520, 1992.

Clegg, S. L., Pitzer, K. S., and Brimblecombe, P.: Thermodynamics of multicomponent, miscible, ionic solutions. II. Mixture including unsymmetrical electrolytes, J. Phys. Chem., 96, 9470-9479, 1992.

Clegg, S. L., Pitzer, K. S., and Brimblecombe, P.: (additions and corrections for their published papers), J. Phys. Chem., 98, 1368, 1994.

Clegg, S. L., Pitzer, K. S., and Brimblecombe, P.: (additions and corrections for their published papers), J. Phys. Chem., 99, 6755, 1995.

Clegg, S. L., Brimblecombe, P., and Wexler, A. S.: Thermodynamic model of the system $\mathrm{H}^{+}-\mathrm{NH}_{4}^{+}-\mathrm{SO}_{4}^{2-}-\mathrm{NO}_{3}^{-}-\mathrm{H}_{2} \mathrm{O}$ at tropospheric temperatures, J. Phys. Chem., A, 102, 2137-2154, 1998a.

Clegg, S. L., Brimblecombe, P., and Wexler, A. S.: Thermodynamic model of the system $\mathrm{H}^{+}-\mathrm{NH}_{4}^{+}-\mathrm{Na}^{+}-\mathrm{SO}_{4}^{2-}-\mathrm{NO}_{3}^{-}-\mathrm{Cl}^{-}-\mathrm{H}_{2} \mathrm{O}$ at $298.15 \mathrm{~K}$, J. Phys. Chem., A, 102, 2155-2171, 1998 b.

Denbigh, K.: The principles of chemical equilibrium, Fourth Ed., Cambridge University Press, Cambridge, 1981.

Dockery, D. W., Pope, C. A., Xu, X., Spengler, J. D., Ware, J. H., Fay, M. E., Ferris, B. G., and Speizer, F. E.: An Association between Air Pollution and Mortality in Six U.S. Cities, The New England Journal of Medicine, 329, 1753-1759, 1993.

Fitzgerald, J. W.: Marine aerosols: A review, Atmos. Environ., 25A, 533-545, 1991. 
Ha, Z. and Chan, C. K.: The water activities of $\mathrm{MgCl}_{2}, \mathrm{Mg}\left(\mathrm{NO}_{3}\right)_{2}$, $\mathrm{MgSO}_{4}$, and their mixtures, Aerosol Sci. Technol., 31, 154-169, 1999.

Heitzenberg, J.: Fine particles in the global troposphere: a review, Tellus, 41B, 149-160, 1989.

Intergovernmental Panel on Climate Change: The scientific Basis, Cambridge Univ. Press, New York, 2001.

Jacobson, M. Z., Tabazadeh, A., and Turco, R. P.: Simulating equilibrium within aerosols and nonequlibrium between gases and aerosols, J. Geophys. Res., 101, 9079-9091, 1996.

Jacobson, M. Z.: Chemical Equilibrium and dissolution processes, Fundamentals of Atmospheric Modeling, Cambridge University Press, New York, pp. 476-510, (Chapter 18), 1999a.

Jacobson, M. Z.: Studying the effect of calcium and magnesium on size-distributed nitrate and ammonium with EQUISOLV II, Atmos. Environ., 33, 3635-3649, 1999 b.

Ramachandran, G. and Vincent, J. H.: A Bayesian approach to retrospective exposure assessment, Applied Occupational and Environmental Hygiene, 14, 547-558, 1999.

Kaiser, J.: Mounting evidence indicts fine-particle pollution, Science, 307, 1858-1861, 2005.

Kelly, J. T. and Wexler, A. S.: Thermodynamics of carbonates and hydrates related to heterogeneous reactions involving mineral aerosol, J. Geophys. Res., 110, D11201, doi:10.1029/2004JD005583, 2005.

Kelly, J. T. and Wexler, A. S.: Water uptake by aerosol: Water activity in supersaturated potassium solutions and deliquescence as a function of temperature, Atmos. Environ, 40, 4450-4468, 2006.

Kim, Y. P., Seinfeld, J. H., and Saxena, P.: Atmospheric gas - aerosol equilibrium I. Thermodynamic model, Aerosol Sci. Technol., 19, 157-181, 1993a.

Kim, Y. P., Seinfeld, J. H., and Saxena, P.: Atmospheric gas aerosol equilibrium II. Analysis of common approximations and activity coefficient calculation methods, Aerosol Sci. Technol., 19, 182-198, 1993b.

Kim, Y. P. and Seinfeld, J. H.: Atmospheric gas - aerosol equilibrium III. Thermodynamics of crustal elements $\mathrm{Ca}^{2+}, \mathrm{K}^{+}$, and $\mathrm{Mg}^{2+}$, Aerosol Sci. Technol., 22, 93-110, 1995.

Kusik, C. L. and Meissner, H. P.: Electrolyte activity coefficients in inorganic processing, AIChE Symp. Series, 173, 14-20, 1978.

Makar, P. A., Bouchet, V. S., and Nenes, A.: Inorganic chemistry calculations using HETV - a vectorized solver for the $\mathrm{SO}_{4}^{2-}-$ $\mathrm{NO}_{3}^{-}-\mathrm{NH}_{4}^{+}$system based on the ISORROPIA algorithms, Atmos. Environ., 37, 2279-2294, 2003.

Meng, Z. Y., Seinfeld, J. H., Saxena, P., and Kim, Y. P.: Atmospheric gas - aerosol equilibrium IV. Thermodynamics of carbonates, Aerosol Sci. Technol., 23, 131-154, 1995.

Meissner, H. P., Kusik, C. L., and Tester, J. W.: Activity coefficients of strong electrolytes in aqueous solution - effect of temperature, AIChE Journal, 18(3), 661-662, 1972.

Meissner, H. P. and Peppas, N. A.: Activity coefficients - aqueous Solutions of polybasic acids and their salts, AIChE Journal, 19(4), 806-809, 1973.

Metzger, S. M., Dentener, F. J., and Lelieveld, J.: Aerosol multiphase chemistry - A parameterization for global modeling, Int. Rep. 99-12, for Mar. and Atmos. Res., Utrecht, Netherlands, 1999.

Metzger, S. M., Dentener, F. J., Lelieveld, J., and Pandis, S. N.: Gas/aerosol partitioning I: a computationally efficient model,
J. Geophys. Res., 107(D16), 4312, doi:10.1029/2001JD001102, 2002a.

Metzger, S. M., Dentener, F. J., Jeuken, A., Krol, M., and Lelieveld, J.: Gas/aerosol partitioning II: global modeling results, J. Geophys. Res., 107(D16), 4313, doi:10.1029/2001JD001103, $2002 \mathrm{~b}$.

Metzger, S., Mihalopoulos, N., and Lelieveld, J.: Importance of mineral cations and organics in gas-aerosol partitioning of reactive nitrogen compounds: case study based on MINOS results, Atmos. Chem. Phys., 6, 2549-2567, 2006,

http://www.atmos-chem-phys.net/6/2549/2006/.

Moya, M., Pandis, S. N., and Jacobson, M. J.: Is the size distribution of urban aerosols determined by thermodynamic equilibrium? An application to Southern California, Atmos. Environ., 36, 2349-2365, 2002.

Moya, M., Ansari, A. S., and Pandis, S. N.: Partitioning of nitrate and ammonium between the gas and particulate phases during the 1997 IMADA-AVER study in Mexico City, Atmos. Environ., 35, 1791-1804, 2001a.

Moya, M., Pandis, S. N., and Jacobson, M. Z.: Is the size distribution of urban aerosols determined by thermodynamic equilibrium? An application to Southern California, Atmos. Environ., 36, 2349-2365, 2001b.

Nenes, A., Pandis, S. N., and Pilinis, C.: ISORROPIA: A new thermodynamic equilibrium model for multiphase multicomponent inorganic aerosols, Aquatic Geochemistry, 4, 123-152, 1998.

Nenes, A., Pilinis, C., and Pandis, S. N.: Continued development and testing of a new thermodynamic aerosol module for urban and regional air quality models, Atmos. Environ., 33, 15531560, 1999.

Pilinis, C. and Seinfeld, J. H.: Continued development of a general equilibrium model for inorganic multicomponent atmospheric aerosols, Atmos. Environ, 21, 2453-2466, 1987.

Pilinis, C. and Seinfeld, J. H.: Water content of atmospheric aerosols, Atmos. Environ., 23, 1601-1606, 1989.

Pitzer, K. S. and Simonson, J. M.: Thermodynamics of multicomponent, miscible, ionic systems: Theory and equations, J. Phys. Chem., 90, 3005-3009, 1986.

Potukuchi, S. and Wexler, A. S.: Identifying solid-aqueous phase transitions in atmospheric aerosols - I. Neutral-acidity solutions, Atmos. Environ., 29, 1663-1676, 1995a.

Potukuchi, S. and Wexler, A. S.: Identifying solid-aqueous phase transitions in atmospheric aerosols - II. Acidic solutions, Atmos. Environ., 29, 3357-3364, 1995 b.

San Martini, F. M., West, J. J., de Foy, B., Molina, L. T., Molina, M. J., Sosa, G., and McRae, G. J.: Modeling inorganic aerosols and their response to changes in precursor concentration in Mexico City, J. Air Waste Manag. Assoc., 55(6), 803-815, 2005.

San Martini, F. M.: Decision Support Tools for Urban Air Quality Management, Ph.D Thesis, Massachusetts Institute of Technology, p. 283, 2004.

Seinfeld, J. H. and Pandis, S. N.: Atmospheric Chemistry and Physics: From Air Pollution to Climate Change, John Wiley \& Sons, Inc., 1998.

Stelson, A. W. and Seinfeld, J. H.: Relative humidity and temperature dependence of the ammonium nitrate dissociation constant, Atmos. Environ., 16(5), 983-992, 1982.

Thomsen, K. and Rasmussen, P.: Modeling of vapor-liquid-solid equilibrium in gas-aqueous electrolyte systems, Chem. Eng. Sci., 54, 1787-1802, 1999. 
Trebs, I., Metzger, S., Meixner, F. X., Helas, G., Hoffer, A., Andreae, M. O., Moura, M. A. L., da Silva, R. S., Slanina, J., Rudich, Y., Falkovich, A., and Artaxo, P.: The $\mathrm{NH}_{4}^{+}-$ $\mathrm{NO}_{3}^{-}-\mathrm{Cl}^{-}-\mathrm{SO}_{4}^{2-}-\mathrm{H}_{2} \mathrm{O}$ system and its gas phase precursors at a rural site in the Amazon Basin: How relevant are crustal species and soluble organic compounds?, J Geophys. Res.-Atmos., 110, D07303, doi:10.1029/2004JD005478, 2005.

Wexler, A. S. and Clegg, S. L.: Atmospheric aerosol models for systems including the ions $\mathrm{H}^{+}, \mathrm{NH}_{4}^{+}, \mathrm{Na}^{+}, \mathrm{SO}_{4}^{2-}$, $\mathrm{NO}_{3}^{-}, \mathrm{Cl}^{-}, \mathrm{Br}^{-}$, and $\mathrm{H}_{2} \mathrm{O}$, J. Geophys. Res., 107, 4207, doi:10.1029/2001JD000451, 2002.

Wexler, A. S. and Seinfeld, J. H.: The distribution of ammonium salts among a size and composition dispersed aerosol, Atmos. Environ., 24A, 1231-1246, 1990.

Wexler, A. S. and Seinfeld, J. H.: Second - generation inorganic aerosol model, Atmos. Environ., 25A, 2731-2748, 1991.

Wexler, A. S. and Clegg, S. L.: Atmospheric aerosol models for systems including the ions $\mathrm{H}^{+}, \mathrm{NH}_{4}^{+}, \mathrm{Na}^{+}, \mathrm{SO}_{4}^{2-}$, $\mathrm{NO}_{3}^{-}, \mathrm{Cl}^{-}, \mathrm{Br}^{-}$, and $\mathrm{H}_{2} \mathrm{O}$, J. Geophys. Res., 107, 4207, doi:10.1029/2001JD000451, 2002.
Yu, S., Dennis. R., Roselle, S., Nenes., A., Walker, J., Eder, B., Schere, K., Swall, J., and Robarge, W.: An assessment of the ability of three-dimensional air quality models with current thermodynamic equilibrium models to predict aerosol $\mathrm{NO}_{3}^{-}$, J. Geophys. Res., 110, D07S13, doi:10.1029/2004JD004718, 2005.

Zanobetti, A., Schwartz, J., and Dockery, D. W.: Airborne particles are a risk factor for hospital admissions for heart and lung disease, Environmental Health Perspective, 108, 1071-1082, 2000.

Zaveri, R. A., Easter, R. C., and Peters, L. K.: A computationally efficient multicomponent equilibrium solver for aerosols (MESA), J. Geophys. Res., 110, D24203, doi:10.1029/2004JD005618, $2005 a$.

Zaveri, R. A., Easter, R. C., and Wexler, A. S.: A new method for multicomponent activity coefficients of electrolytes in aqueous atmospheric aerosols, J. Geophys. Res., 110, D02201, doi:10.1029/2004JD004681, 2005b.

Zhang, Y., Seigneur, C., Seinfeld, J. H., Jacobson, M., Clegg, S. L., and Binkowski, F. S.: A comparative review of inorganic aerosol thermodynamic equilibrium models: similarities, differences, and their likely causes, Atmos. Environ., 34, 117-137, 2000 . 